\title{
Dephasing in sequential tunneling through a double-dot interferometer
}

\author{
Florian Marquardt* and C. Bruder \\ Departement Physik und Astronomie, Universität Basel, Klingelbergstrasse 82, CH-4056 Basel, Switzerland
}

(Received 22 March 2003; revised manuscript received 19 June 2003; published 5 November 2003)

\begin{abstract}
We analyze dephasing in a model system where electrons tunnel sequentially through a symmetric interference setup consisting of two single-level quantum dots. Depending on the phase difference between the two tunneling paths, this may result in perfect destructive interference. However, if the dots are coupled to a bath, it may act as a which-way detector, leading to partial suppression of the phase coherence and the reappearance of a finite tunneling current. In our approach, the tunneling is treated in leading order whereas coupling to the bath is kept to all orders [using $P(E)$ theory]. We discuss the influence of different bath spectra on the visibility of the interference pattern, including the distinction between "mere renormalization effects" and "true dephasing."
\end{abstract}

DOI: $10.1103 /$ PhysRevB.68.195305

PACS number(s): 73.23.Hk, 71.38.-k, 03.65.Yz

\section{INTRODUCTION}

The destruction of quantum-mechanical phase coherence due to coupling of a system to an irreversible bath is a subject important not only because of its connection to fundamental issues (the quantum measurement process and the quantum-classical transition) but also because of its role in the suppression of phenomena resulting from quantum interference effects, such as those studied in mesoscopic physics (including Aharonov-Bohm interference, weak localization, and universal conductance fluctuations). Recently, the field of mesoscopic physics in particular has seen a revival of interest in these questions, due to surprising experimental findings ${ }^{1}$ concerning a possible saturation of the weaklocalization dephasing rate at low temperatures, which have not yet been explained convincingly. Apart from investigations dealing directly with the problem of weak localization in a disordered system of interacting electrons, several toy models have been analyzed ${ }^{2-9}$ to answer the question whether decoherence at zero temperature is possible at all, contrary to the expectations based on perturbation theory. One of the difficulties faced by models involving discrete levels consists in the fact that destruction of phase coherence for a superposition of excited states of finite excitation energy is perfectly possible even at zero temperature, due to spontaneous emission of energy into the bath. It is only in the zero-frequency limit of the linear response in a system with a continuous spectrum (relevant for weak localization and other equilibrium transport experiments) that perturbation theory suggests in general a vanishing dephasing rate, because then the perturbation does not supply energy to the system, such that at $T=0$ the system is not able to leave a trace in the bath, which is considered to be the prerequisite for decoherence.

Some questions of interest concerning dephasing, especially in connection with mesoscopic systems and low temperatures, are the following ones. How reliable is the simple classical picture of a phase being randomized by fluctuating external noise ${ }^{10}$ In particular, what is the meaning of the zero-point fluctuations of the bath in this picture, as opposed to the thermal fluctuations dominating at frequencies lower than the temperature? When do the former lead to "mere renormalization effects" and how is it possible to distinguish these from "true" dephasing? Under which circumstances is the suppression of off-diagonal terms in the reduced system density matrix itself already a good indicator of dephasing? How reliable are simple arguments based on golden rule and energy conservation, related to the connection between dephasing and the trace left in the bath by the particle ("which-way" detection)? When does perturbation theory fail qualitatively, what is the influence of non-Markoffian behavior? How does the dephasing rate depend on the energy supplied by an external perturbation (frequencies excited in linear response, bias voltage applied in a transport measurement)? What is the influence of the Pauli principle in a system of degenerate fermions? How strong are the qualitative differences in behavior resulting from different bath spectra?

In this work, we will present a model that is able to give insights into most of these questions.

Our model represents a kind of mesoscopic double-slit setup. It consists of two single-level quantum dots which are tunnel coupled to two leads, with a possible phase difference between the two interfering paths (see Fig. 1). Due to destructive interference (at $\varphi=\pi$ ), the tunneling current may be suppressed completely, provided the two dot levels are degenerate and the setup is symmetric in the two interfering paths. Coupling the dots to a bath may partly destroy the phase coherence and reenable the electrons to go through the

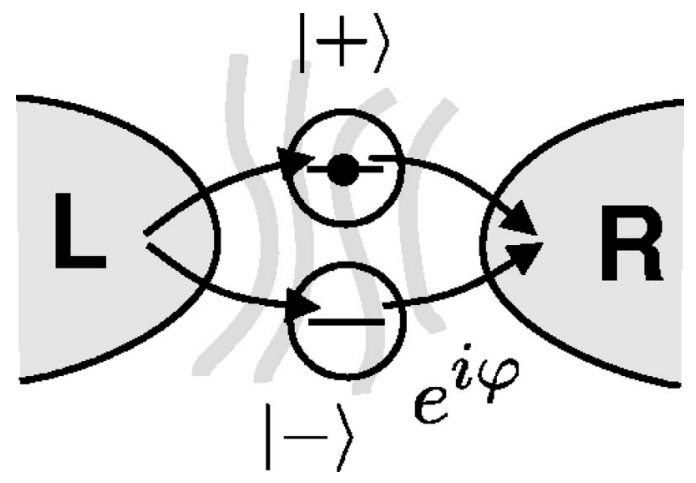

FIG. 1. The double-dot "double-slit" setup, with a fixed phase difference $\varphi$ between the two paths and under the influence of a fluctuating environment. 
setup. For a symmetric setup, with equal coupling strength between the bath and each of the two dots, mere renormalization effects will not be able to lift the destructive interference in this way. Thus, a finite tunneling current may be taken as a genuine sign of dephasing. This criterion for dephasing has been employed before in a model of dephasing due to spin-flip transitions in first-order tunneling transport through one or two dots, ${ }^{11}$ as well as for cotunneling through an Aharonov-Bohm ring coupled to a fluctuating magnetic flux. ${ }^{5}$

The influence of phonons on sequential tunneling through two quantum dots in series has been studied experimentally in Ref. 12. There, inelastic transitions induced by piezoelectric coupling to acoustic phonons in GaAs have been essential for obtaining a finite current through the two off-resonant dot levels. This kind of setup has been analyzed theoretically in Refs. 13-19. On the other hand, we will be analyzing tunneling through two dots placed in parallel. Early theoretical investigations of this problem (without a fluctuating environment) include Refs. 20 and 21. Recently, a parallel-dot tunneling setup has been realized experimentally in Ref. 22, with an emphasis on spectroscopy of the "molecular states" of the doubledot system (with interdot tunneling present). In our model of an interference setup, we choose to describe a situation without tunneling between the dots (but with Coulomb repulsion). In addition, we want to concentrate on interference effects in the orbital motion and therefore consider the case of spin-polarized transport. This model-in the absence of a fluctuating environment-has been investigated previously in Ref. 23. Other recent theoretical works concerning tunneling through dots in a parallel geometry have mostly investigated spin and Kondo physics, ${ }^{24-27}$ but also dephasing by spin-flip transitions. ${ }^{11}$ Some works have treated the influence of phonons in tunneling interference structures, ${ }^{28,29}$ but no systematic discussion of dephasing and the visibility of the interference pattern had been given. Some while ago, dephasing by nonequilibrium current noise has been investigated experimentally ${ }^{30}$ and theoretically ${ }^{31}$ in a setup with a single quantum dot placed into one arm of an Aharonov-Bohm interferometer.

Our analysis of dephasing in sequential tunneling through a double dot will take into account the system-bath coupling exactly, while we treat the tunnel coupling only in leading order. The presence of the Fermi sea in the leads introduces some aspects related to the Pauli principle and to the behavior of systems with a continuous spectrum that cannot be analyzed in simpler models of dephasing in discrete systems coupled to a bath.

The work is organized as follows: After setting up the model (Sec. II), we will present a qualitative discussion of its main features (Sec. III). In particular, we will discuss the relation between entanglement, dephasing, and renormalization effects. Subsequently, we derive a general formula for the tunneling decay rate of an electron that has been placed on the two dots in a symmetric superposition of states (Sec. IV). This is done by building on the concepts of the $P(E)$ theory of tunneling in a dissipative environment. ${ }^{32,33}$ Following this, we will evaluate the dependence of the tunneling rate on the bias voltage and the bath spectra (Sec. V). We will interpret the results in terms of "renormalization effects" and "true dephasing" (Sec. VI). Building on these sections, we will finally derive a master equation for the case of weak tunnel coupling (Sec. VII), which allows us to calculate the sequential tunneling current as a function of bias voltage, temperature, and phase difference (Sec. VIII).

The most important results derived in this work are the following. Equation (13) is the general expression for the phase-dependent tunneling decay rate in presence of the fluctuating environment. It forms the basic input for the master equation [Eqs. (48)-(50)], which describes sequential tunneling through the double dot, where the resulting current can be obtained from Eq. (52). The visibility of the interference pattern, which is defined by the phase dependence of the current, is given in Eq. (65). It is connected with the visibility obtained from the phase dependence of the tunneling rate itself [Eqs. (16) and (17)].

\section{THE MODEL}

We consider a Hamiltonian describing two degenerate single-level quantum dots, with respective single-particle states $|+\rangle$ and $|-\rangle$ (spin is excluded for simplicity, since we are interested in dephasing of the electronic motion). Each of them is tunnel coupled to two electrodes (with the same strength for both dots), but involving a possible phase difference between the tunnel amplitudes (see Fig. 1). In addition, the potential difference between the two dots is given by a fluctuating field $\hat{F}$, whose dynamics is derived from a linear bath. It represents the fluctuations due to phonons or Nyquist noise. The system-bath coupling strength is taken to be the same for both dots, while the sign is opposite, such that the bath can distinguish between an electron being on dot $|+\rangle$ or $|-\rangle$ :

$$
\begin{aligned}
\hat{H}= & \boldsymbol{\epsilon}\left(\hat{n}_{+}+\hat{n}_{-}\right)+\hat{F}\left(\hat{n}_{+}-\hat{n}_{-}\right)+U \hat{n}_{+} \hat{n}_{-} \\
& +\hat{H}_{L}+\hat{H}_{R}+\hat{H}_{B}+\hat{V} .
\end{aligned}
$$

Here $\hat{n}_{ \pm}$are the particle numbers on the two dots (equal to 0 or 1 ). The bath Hamiltonian $\hat{H}_{B}$ describes a set of uncoupled harmonic oscillators. It governs the dynamics of the fluctuating potential $\hat{F}$, which is assumed to be linear in the oscillator coordinates. The coupling between electron and bath is of the form of the "independent boson model." ${ }^{34}$ For the case of exactly one electron on the double dot, and in the absence of tunneling, it corresponds to a spin-boson model with "diagonal coupling." In this model, no transition between different levels is brought about by the bath, such that pure dephasing results. $U$ denotes the Coulomb repulsion energy, which we will take to be so large that double occupancy is forbidden. Note that the degeneracy of the two dot levels is important in the following: It is necessary to ensure complete destructive interference at $\varphi=\pi$ (compare also the discussion in Sec. VII).

The terms $\hat{H}_{L}$ and $\hat{H}_{R}$ contain the energies of the electrons in the left and right reservoirs: 


$$
\hat{H}_{L(R)}=\sum_{k} \epsilon_{k} \hat{a}_{L(R) k}^{\dagger} \hat{a}_{L(R) k} .
$$

The tunneling between the dots and the leads is described by $\hat{V}=\hat{V}_{L}+\hat{V}_{R}$, with

$$
\hat{V}_{R}=\sum_{k} t_{k}^{R} \hat{a}_{R k}^{\dagger}\left(\hat{d}_{+}+e^{i \varphi} \hat{d}_{-}\right)+\text {H.c. }
$$

for the right junction, and

$$
\hat{V}_{L}=\sum_{k} t_{k}^{L} \hat{a}_{L k}^{\dagger}\left(\hat{d}_{+}+\hat{d}_{-}\right)+\text {H.c. }
$$

for the left junction.

Here $\hat{d}_{ \pm}$are the annihilation operators for the two dots $\left(\hat{n}_{ \pm}=\hat{d}_{ \pm}^{\dagger} \hat{d}_{ \pm}\right)$and the phase factor of $e^{i \varphi}$ controls the interference between tunneling events along either the upper or lower path. The tunneling phase difference might be thought of as arising due to the Aharonov-Bohm phase from a magnetic flux penetrating the region between the quantum dots.

Note that the tunneling matrix elements $t_{k}^{R(L)}$ are assumed not to depend on the dot state $|+\rangle$ or $|-\rangle$ in our model. This means that the dots are close enough such that they couple to the same point on the lead electrodes, to within less than a Fermi wavelength. Obviously, there could be no appreciable interference effect if the dots were separated by some larger distance (in which case the $k$ dependence of matrix elements would be different for the two states). The same idealized assumption underlies several similar models (see, e.g., Refs. $11,23,27)$. The effect of an arbitrary dot separation has been discussed in some detail in Ref. 21.

The present model, without the bath, has been analyzed previously in Ref. 23 (see also Sec. IV C of Ref. 11). There, an orbital type of Kondo effect was found in equilibrium, for $\varphi=\pi$, when the level energy was below the chemical potential. This arises because at $\varphi=\pi$ there are two states of the double dot that couple only to the left and the right lead, respectively (denoted by $|e\rangle$ and $|o\rangle$ in the following). These degenerate states form the pseudospin responsible for the Kondo effect. However, that mechanism will be irrelevant for our analysis, as we consider the transport situation where the (renormalized) level energy lies between the chemical potentials of the left and the right lead. Therefore, the degeneracy is effectively lifted by the bias voltage (which will be assumed to be much larger than the tunneling rate), and only the state coupling to the left lead would be occupied at $\varphi=\pi$.

\section{QUALITATIVE DISCUSSION}

In this and the following three sections, we first analyze the escape of a single electron into the right lead, where the electron is assumed to start out in a symmetric superposition of the two dot levels, which has been formed by an electron tunneling onto the dots from the left lead. In the situation without any bath, this is the state $|e\rangle \equiv(|+\rangle+|-\rangle) / \sqrt{2}$.

Without dephasing, the tunneling decay out of state $|e\rangle$ is made impossible in the case of perfect destructive interfer-

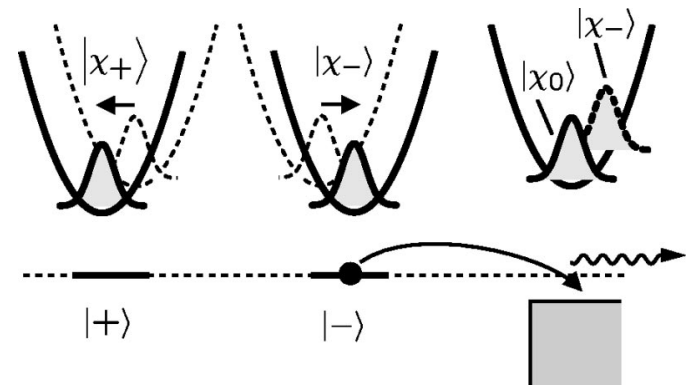

FIG. 2. The ground state $\left|\chi_{+}\right\rangle\left(\left|\chi_{-}\right\rangle\right)$which the bath assumes in the presence of an electron on dot $|+\rangle(|-\rangle)$, shown schematically for a single bath oscillator (see main text). After the electron has tunneled into the lead, $\left|\chi_{-}\right\rangle$becomes a superposition of excited states (dashed curve), while the state $\left|\chi_{0}\right\rangle$ represents the ground state of the bath in the new potential.

ence at $\varphi=\pi$, while maximal constructive interference is present for $\varphi=0$. It should be noted that the attribution of the phase factor to one of the tunnel couplings represents a certain choice of gauge, which affects the wave functions in the following discussion but none of the physically observable quantities that are derived as a result of the master equation in Sec. VII.

For simplicity, we will assume a zero-temperature situation throughout the following qualitative discussion, with a bias $e V>0$ applied between the two dots and the lead in such a way that the electron is allowed to tunnel into the lead (see Fig. 2). In addition, since we will describe the tunneling decay to the right, we will only consider the coupling $\hat{V}_{R}$ to the right lead in this section and drop the index $R$ for now.

Without the bath and for perfect constructive interference $(\varphi=0)$, the tunneling decay rate $\Gamma$ will take on its maximum value of $2 \Gamma_{0}$, with

$$
\Gamma_{0} \equiv 2 \pi D\left\langle\left|t_{k}\right|^{2}\right\rangle
$$

where $D$ is the lead density of states at the Fermi energy, $\left\langle\left|t_{k}\right|^{2}\right\rangle$ is the angular average of $\left|t_{k}\right|^{2}$ at this energy. The bias voltage $V$ does not enter in this case, as long as it is positive (permitting decay). For $\varphi=\pi, \Gamma$ vanishes due to perfect destructive interference. In general, we have

$$
\Gamma=\Gamma_{0}(1+\cos \varphi) .
$$

If the bath is included in the description, the following happens.

First of all, the energy of a single extra electron on any of the two dots will be renormalized from its initial value of $\epsilon$, since the bath relaxes to a ground state of lower energy in presence of the electron. We will assume that the value of $\epsilon$ has been chosen exactly to compensate for this energy change, which is given by $-\int_{0}^{\infty} d \omega\langle\hat{F} \hat{F}\rangle_{\omega} / \omega$ (see Appendix A). Then, the energy of an electron on the dot (and the bath in its new ground state) is the same as that of the electron being in the lead, at the Fermi energy of $\epsilon_{F} \equiv 0$ (for $V=0$ ).

Tunneling of an electron from the dots to the lead will not change the bath state, but it will displace the origin of the harmonic oscillators comprising the bath, since the coupling 
to $\hat{F}$ is switched off ( $\hat{n}_{+}-\hat{n}_{-}$changes to zero). Therefore, the original ground state of the bath (in presence of the electron) will become a superposition of excited states in the new bath potential (in absence of the electron; see Fig. 2). On the other hand, since energy conservation has to be fulfilled with respect to the total energy of the electrons and the bath before and after the tunneling event, only those excited bath states can be reached whose energies are not greater than $e V$, the energy supplied to the electron by the bias voltage. This leads to the Coulomb-blockade type suppression of the tunneling rate at low bias voltages, for $\varphi=0$. Physically, this effect is just the same as that described by Franck-Condon overlap integrals evaluated between vibronic states for electronic transitions in molecules. Qualitatively, this effect is independent of the interference setup, since it already occurs for tunneling through a single dot coupled to a bath.

In contrast, for the case of destructive interference $(\varphi$ $=\pi$ ), the bath may actually enhance the tunneling rate from its initial value of 0 , since it partly destroys the phase coherence that is a presupposition for perfect interference. An electron coming from the left lead will form the following entangled state with the bath, instead of the symmetric superposition $|e\rangle=(|+\rangle+|-\rangle) / \sqrt{2}$ :

$$
\left(|+\rangle\left|\chi_{+}\right\rangle+|-\rangle\left|\chi_{-}\right\rangle\right) / \sqrt{2}
$$

Here the states $\left|\chi_{ \pm}\right\rangle$denote the respective ground states of the bath for a bath Hamiltonian given by $\hat{H}_{B} \pm \hat{F}$, which are related to each other by a parity transformation. (This also means we assume by definition there to be no phase factor between these states; e.g., both may be assumed to have real-valued positive wave functions.) Actually, the entangled state considered here will be formed only if the electron is given barely enough energy to enter the double dot at all (i.e., chemical potential of the left lead infinitesimally larger than the renormalized level position). Otherwise, excited bath states may be created even at this step. These complications will be taken care of in the complete discussion of the sequential tunneling current (Sec. VII). There, it will turn out that the tunneling decay rate derived in the following, based on our physically motivated ansatz (7), is exactly the rate that enters the full master equation. Thus, we proceed with ansatz (7) for the initial entangled state, in order to calculate the rate for such an electron to tunnel into the right lead.

The bath measures (to some extent) which dot the electron resides on, such that the reduced system density matrix (for the electron on the two dots) becomes mixed and its off-diagonal elements get suppressed by the overlap factor $\left\langle\chi_{+} \mid \chi_{-}\right\rangle$. Put differently, the phase factor between the two dot states in the wave function of the electron (initially equal to +1 ) becomes uncertain. Therefore, there is a finite probability of

$$
P_{o}=\left(1-\left\langle\chi_{+} \mid \chi_{-}\right\rangle\right) / 2
$$

to find the electron in the antisymmetric (odd) state $|o\rangle \equiv$ $(|+\rangle-|-\rangle) / \sqrt{2}$. At $\varphi=\pi$, where tunneling decay of the symmetric superposition $|e\rangle$ is blocked due to destructive interference, the state $|o\rangle$ is allowed to decay into the lead, at the maximal rate of $2 \Gamma_{0}$. In this way, the interferenceinduced blockade of electron tunneling is lifted by dephasing.

However, this simple picture is true only for large bias voltages, when energy conservation permits any final state of the bath after the tunneling event. If the maximum energy supplied to the electron is limited, the suppression discussed above (for the case of $\varphi=0$ ) will apply again. In particular, if the bias voltage is turned to zero, energy conservation only allows the state $\left|\chi_{0}\right\rangle$ to be reached, which is the ground state of the bath in the absence of any electrons on the dots. Then, the tunneling rate is exactly zero again, despite the fact that the reduced density matrix of the electron may be mixed to a strong extent. The reason is the following. When the overlap of the entangled state (7) with the state $\left|\chi_{0}\right\rangle$ is taken, the two overlap factors $\left\langle\chi_{0} \mid \chi_{+}\right\rangle$and $\left\langle\chi_{0} \mid \chi_{-}\right\rangle$turn out to be the same, if the coupling of the bath to the two dots is symmetric (i.e., of equal strength, only of opposite sign), which we have assumed in writing down the Hamiltonian, Eq. (1). Therefore, the electronic state resulting from the projection of Eq. (7) onto $\left|\chi_{0}\right\rangle$ is equal to the symmetric combination, whose decay is forbidden. Thus, the combination of energy conservation and Pauli blocking prevents a finite tunneling rate at zero bias voltage, in spite of the mixed state of the electron coupled to the bath. In this limit the entanglement between electron and bath only leads to renormalization effects (such as the change in tunneling rate), but not to genuine dephasing. If the coupling were asymmetric, then destructive interference could be lost even without dephasing (merely due to renormalization), just as it would be the case for initially asymmetric bare tunnel couplings. That is why the asymmetric case is uninteresting for our purposes of distinguishing renormalization effects from real dephasing.

However, whether we are indeed able to claim that dephasing actually vanishes in the limit of low bias voltages will depend on the behavior of the tunneling rate as a function of $V$ and on the comparison of the cases $\varphi=0$ and $\varphi$ $=\pi$. Here, the bath spectrum, and, above all, its lowfrequency properties, enter. In order to be able to discuss $\Gamma(V, \varphi)$ quantitatively, we will make use of the concepts of the $P(E)$ theory of tunneling in a dissipative environment.

\section{DECAY RATE AND CONNECTION TO $P(E)$ THEORY}

The tunneling rate $\Gamma$ will be calculated using the standard Fermi golden rule, i.e., lowest-order perturbation theory in the bare tunneling rate $\Gamma_{0}$, but taking into account exactly the bath coupling. In deriving the formula for $\Gamma$, it turns out to be useful to assume that the bath oscillators do not get shifted in the tunneling event (unlike the qualitative considerations from above), but it is rather the bath states which get displaced (in the opposite direction). Obviously, this amounts to the same, as long as we are interested only in overlap integrals of different bath states after the event. To that end, we introduce the displacement operator $\exp (i \hat{\phi})$, which transforms the bath ground state of $\hat{H}_{B}$ into that of $\hat{H}_{B}+\hat{F}$. Here $\hat{\phi}$ is a suitable Hermitian operator that is linear in the 
bosonic variables of the bath. In fact, this amounts to performing the canonical transformation of the independent boson model,${ }^{34}$ see Appendix A. In terms of the two dot states + and - , we have $\hat{F}_{+}=\hat{F}$ and $\hat{F}_{-}=-\hat{F}$, as well as $\hat{\phi}_{+}$ $=\hat{\phi}$ and $\hat{\phi}_{-}=-\hat{\phi}$. The transformation eliminates the system-bath coupling from the Hamiltonian, but gives rise to modified dot operators $\hat{d}_{ \pm}^{\prime}=e^{ \pm i} \hat{\phi} \hat{d}_{ \pm}$in the transformed tunnel Hamiltonian $\hat{V}_{R}^{\prime}$ [see Eq. (A7)].

We will assume the tunnel coupling to be sufficiently weak, such that we can use lowest-order perturbation theory to calculate the tunneling decay rate

$$
\Gamma=2 \pi \sum_{f}\left|\left\langle f\left|\hat{V}_{R}^{\prime}\right| i\right\rangle\right|^{2} \delta\left(E_{f}-E_{i}\right),
$$

where the initial state $|i\rangle$ is given by the configuration involving the electron residing in the symmetric superposition on the dots, the unperturbed Fermi sea in the lead, and the bath in its ground state $\left|i_{B}\right\rangle$. The bath ground state has become independent of the position of the electron, due to the above-mentioned transformation. At finite temperatures, an additional thermal average over the initial bath state and the initial state of the electrons in the lead has to be performed. The energies and eigenstates refer to the Hamiltonian without tunnel coupling. Applying the new tunneling Hamiltonian $\hat{V}_{R}^{\prime}$ to the initial state, we obtain the following expression:

$$
\begin{aligned}
\Gamma= & \pi \sum_{k, f_{B}}\left|t_{k}\right|^{2}\left[1-f\left(\epsilon_{k}+e V\right)\right] \mid \\
& \times\left.\left\langle f_{B}\left|e^{+i \hat{\phi}}+e^{i \varphi} e^{-i \hat{\phi}}\right| i_{B}\right\rangle\right|^{2} \delta\left(E_{f}^{B}-E_{i}^{B}+\epsilon_{k}\right) .
\end{aligned}
$$

Here $f(\cdot)$ is the Fermi function (for chemical potential equal to zero), and $E_{f, i}^{B}$ are the energies of the initial and final bath states. The energy supplied to the bath is equal to the energy lost by the electron (given by $-\epsilon_{k}$, since the renormalized dot energy is zero). Following the usual derivation of the $P(E)$ theory, ${ }^{32,33}$ we express the energy-conserving $\delta$ function as an integral over time and also replace the sum over lead states $k$ by an integral over the energy $E=-\epsilon_{k}$ supplied to the bath, finally yielding

$$
\begin{aligned}
\Gamma= & \Gamma_{0} \int_{-\infty}^{+\infty} d E[1-f(-E+e V)] \int_{-\infty}^{+\infty} \frac{d t}{2 \pi} e^{i E t} \\
& \times \frac{1}{2}\left\langle\left(e^{-i \hat{\phi}(t)}+e^{-i \varphi} e^{i \hat{\phi}(t)}\right)\left(e^{i \hat{\phi}}+e^{i \varphi} e^{-i \hat{\phi}}\right)\right\rangle .
\end{aligned}
$$

For the case of arbitrary temperature, the brackets denote a thermal average over the initial bath state $\left|i_{B}\right\rangle$. We introduce the definitions

$$
P_{(-)}(E)=\frac{1}{2 \pi} \int_{-\infty}^{+\infty} d t e^{i E t} e^{ \pm\langle\hat{\phi}(t) \hat{\phi}\rangle-\left\langle\hat{\phi}^{2}\right\rangle} .
$$

This permits us to write down our final result for the tunneling decay rate in terms of $P_{(-)}(E)$,

$$
\Gamma=\Gamma_{0} \int_{-\infty}^{+\infty} d E[1-f(-E+e V)]\left[P(E)+\cos (\varphi) P_{-}(E)\right]
$$

The formula given here constitutes the basic expression for the decay rate as a function of bias voltage and interference phase $\varphi$. It represents the appropriate modification of Eq. (6) in presence of a bath.

Note that for the slightly more general case of arbitrarily correlated fluctuating potentials $\hat{F}_{+}$and $\hat{F}_{-}$attached to the dots (i.e., an interaction of the form $\hat{F}_{+} \hat{n}_{+}+\hat{F}_{-} \hat{n}_{-}$), the function $P_{-}(E)$ would contain the cross correlator of the associated phases $\hat{\phi}_{+}$and $\hat{\phi}_{-}$, while $P(E)$ would depend on the autocorrelator of $\hat{\phi}_{+}$or $\hat{\phi}_{-}$(assumed to be the same, for the setup to remain symmetric). In contrast to the model treated here, such an interaction would also involve fluctuations of the sum of energies of the dot levels. However, they would only add to the renormalization effects mentioned previously and do not contribute to dephasing by themselves, since such fluctuations cannot distinguish between the two interfering paths.

By using the definitions

$$
\gamma_{(-)} \equiv \Gamma_{0} \int d E[1-f(-E+e V)] P_{(-)}(E),
$$

we can write

$$
\Gamma=\gamma+\cos (\varphi) \gamma_{-} .
$$

The strength of the dependence of $\Gamma$ on the phase $\varphi$ may be taken as a signature of phase coherence in our model. We define the "visibility" of the interference pattern in the usual way, by

$$
v \equiv\left(\Gamma_{\max }-\Gamma_{\min }\right) /\left(\Gamma_{\max }+\Gamma_{\min }\right),
$$

which is equal to the ratio

$$
v=\frac{\gamma_{-}}{\gamma}
$$

The visibility $v$ will be 1 whenever the destructive interference is perfect, and it is zero if there is no dependence of $\Gamma$ on $\varphi$.

The effects of the bath on the decay rate are encoded in the functions $P(E)$ and $P_{-}(E)$, whose general properties we will discuss now. In the following section, we will evaluate them for different types of bath spectra.

As usual, the function $P(E)$ describes the probability (density) that an electron will emit the energy $E$ into the bath while tunneling into the lead. It is real, non-negative, and normalized to unity. ${ }^{33,32}$

At large times $|t| \rightarrow \infty$, the correlation function $\langle\hat{\phi}(t) \hat{\phi}\rangle$ $-\left\langle\hat{\phi}^{2}\right\rangle$ in the exponent of integral (12) will tend to $-\left\langle\hat{\phi}^{2}\right\rangle$, for a continuous bath spectrum. This means that the integrand of $P(E)$ approaches the value of $z \equiv \exp \left(-\left\langle\hat{\phi}^{2}\right\rangle\right)$, starting from 1 at $t=0$. Therefore, $P(E)$ contains a "quasiparticle $\delta$ peak" of strength $z$ at $E=0$, if $z$ does not vanish. 
It corresponds to the probability $z$ of having no energy transfer at all from the electron to the bath (similar to the recoilfree emission of a $\gamma$ ray by a nucleus inside a crystal, i.e., the Mössbauer effect).

The function $P_{-}(E)$ in front of the $\cos (\varphi)$ term in Eq. (13) is different: The integrand of $P_{-}(E)$ will increase at large times, towards the value of $z$, starting from $z^{2}$ at $t$ $=0$. The function $P_{-}(E)$ is real valued [because of $\left.\langle\hat{\phi}(t) \hat{\phi}\rangle=\langle\hat{\phi} \hat{\phi}(t)\rangle^{*}\right]$, but it can become negative. Therefore, it cannot be interpreted as a probability density, in contrast to $P(E)$. Its normalization is given by

$$
\int d E P_{-}(E)=z^{2}
$$

If $z$ is nonzero, $P_{-}(E)$ also has a $\delta$ peak at $E=0$, of weight $z$, just as $P(E)$. As a consequence, in the case of destructive interference $(\varphi=\pi)$, the tunneling rate $\Gamma$ at $V \rightarrow 0, T=0$ still vanishes even in the presence of the bath, since the $\delta$ peaks contained in $P(E)$ and $P_{-}(E)$ cancel exactly in integral (13). The physical reason for this coherence has been discussed at the end of the preceding section.

In the case of constructive interference $(\varphi=0)$, at $T=0$ and for $V \rightarrow 0$, the integration over $E$ will only capture the $\delta$ peaks contained in $P_{(-)}(E)$, yielding $\Gamma=2 z \Gamma_{0}$. Thus, the tunneling rate is suppressed by the constant factor $z$ from its noninteracting value. However, this may be interpreted as a mere renormalization of the effective tunnel coupling, since the visibility $v$ of the interference pattern is still equal to unity. In order to connect this result to the qualitative discussion from above, we note that the overlap of the two different bath ground states that are adapted to the absence or presence of an electron on dot \pm , is given by

$$
\left\langle\chi_{0} \mid \chi_{ \pm}\right\rangle=\left\langle\chi_{0}\left|e^{ \pm i \hat{\phi}}\right| \chi_{0}\right\rangle=\exp \left(-\left\langle\hat{\phi}^{2}\right\rangle / 2\right)=z^{1 / 2} .
$$

Therefore, the magnitude squared of this overlap, which determines the probability of tunneling without exciting any bath mode, is equal to $z$.

On the other hand, for sufficiently large bias voltages (much larger than the cutoff frequency of the bath spectrum), the normalization conditions for $P_{(-)}(E)$ yield

$$
\Gamma=\Gamma_{0}\left[1+z^{2} \cos (\varphi)\right] .
$$

The visibility is given by $v=z^{2}$. In this limiting case, where the restrictions due to energy conservation and the Pauli principle are no longer important, the tunneling rate $\Gamma$ at the point $\varphi=\pi$ of destructive interference does not vanish. It takes the value $\Gamma_{0}\left(1-z^{2}\right)$, which is small if the effects of the bath are weak ( $z$ near to 1 ) and is equal to one half the ideal maximum value $2 \Gamma_{0}$ for a bath that is sufficiently strong to destroy phase coherence completely $(z=0)$, leading to an incoherent mixture of symmetric and antisymmetric states on the two dots. In the latter case, the visibility vanishes (even for arbitrary voltages), since then $P_{-}(E)$ is equal to zero, which makes $\Gamma$ independent of $\varphi$. This will be true for the Ohmic bath, to be discussed in the following section.

As explained above, the reduced density matrix of the electron on the dots coupled to the bath predicts a finite probability of $P_{o}=\left(1-\left\langle\chi_{+} \mid \chi_{-}\right\rangle\right) / 2$ to find the electron in the antisymmetric state if one starts out from the symmetric superposition before coupling it to the bath. The overlap factor of the bath states involved in this probability can be expressed as

$$
\left\langle\chi_{+} \mid \chi_{-}\right\rangle=\left\langle\chi_{0}\left|\left(e^{-i \hat{\phi}}\right)^{2}\right| \chi_{0}\right\rangle=z^{2}
$$

Comparing with the result $\Gamma(\varphi=\pi)=\Gamma_{0}\left(1-z^{2}\right)$ given above, it may be observed that the decay rate at sufficiently large bias voltages is indeed determined directly by the probability to find the electron in the state whose decay is not forbidden by destructive interference [as has been argued already at the end of the preceding section, near Eq. (8)]. It is only in this limiting case, where an arbitrary amount of energy is available for excitation of the bath, that the suppression of interference effects in the transport situation is correctly deduced from the electron's reduced density matrix in the presence of the bath. Formally, this holds because the sum over final bath states $f_{B}$ in Eq. (10) is not restricted any more and corresponds to the insertion of a complete set of basis states. Thus, one obtains, directly from Eq. (10),

$$
\Gamma=\frac{\Gamma_{0}}{2}\left\langle\chi_{+}+e^{-i \varphi} \chi_{-} \mid \chi_{+}+e^{i \varphi} \chi_{-}\right\rangle,
$$

which reduces to Eq. (20) when the overlaps are evaluated, using Eq. (21). Physically, the case of high bias voltage corresponds to a kind of infinitely fast von Neumann projection measurement that determines the state of the electron, revealing the fluctuations due to the bath. In contrast, at low bias voltages (low energy supply), a kind of "weak" measurement is carried out that takes a longer amount of time, such that only the low-frequency fluctuations of the bath are important for dephasing.

\section{EVALUATION FOR DIFFERENT BATH SPECTRA}

We will restrict the discussion to $T=0$ at first.

The simplest example for the bath is a single harmonic oscillator of frequency $\omega$. This offers an approximate description of the interaction with optical phonon modes ("Einstein model"). In this case, $P(E)$ and $P_{-}(E)$ can be obtained easily by expanding the exponential in a Taylor series and using $\langle\hat{\phi}(t) \hat{\phi}\rangle=\left\langle\hat{\phi}^{2}\right\rangle \exp (-i \omega t)$, before the integration over time is performed. For $P(E)$, the resulting series of $\delta$ peaks at harmonics of $\omega$ corresponds to all possible processes where the electron emits any number $n$ of phonons into the bath while tunneling into the lead. The expression for $P_{-}(E)$ is the same, apart from alternating signs in front of the $\delta$ functions: 


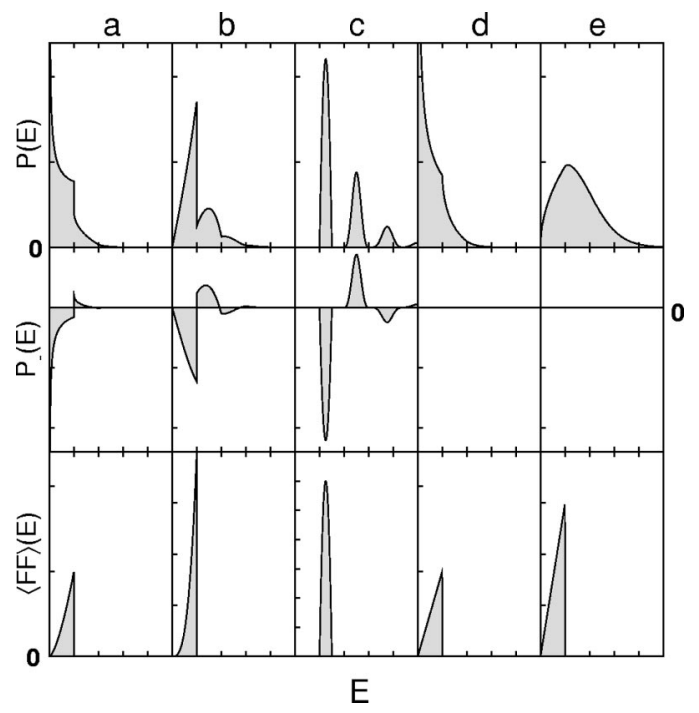

FIG. 3. The bath spectrum $\langle\hat{F} \hat{F}\rangle_{E}$ (bottom) and the resulting functions $P(E)$ (top) and $P_{-}(E)$ (middle), plotted vs energy $E$, for different baths. Energies are measured in units of the "bath cutoff" $\omega_{c}$. Energy axis is the same in all panels (starting at $E=0$, horizontal tick distance: 1 ); vertical tick distance in all panels is 0.5 . a, $s=1.5, \alpha=0.25$; b, "acoustic phonons," $s=3, \alpha=1$; c, "optical phonons," Bath with gap; d, $s=1, \alpha=0.25$; e, $s=1, \alpha=0.75$ (d, e are "Ohmic" baths of different strength, $z=0$ ).

$$
P_{(-)}(E)=z \sum_{n=0}^{\infty} \frac{\left\langle \pm \hat{\phi}^{2}\right\rangle^{n}}{n !} \delta(E-n \omega)
$$

Thus, every process involving the transfer of an even number of quanta to the bath will not ruin the destructive interference at $\varphi=\pi$, since the corresponding contributions from $P(E)$ and $P_{-}(E)$ cancel in Eq. (13). This is because the coupling between electron and bath is of the type $\left(\hat{n}_{+}-\hat{n}_{-}\right) \hat{F}$, which gives a different sign of the interaction amplitude for a phonon emission process, depending on the dot. Therefore, the amplitude of emission of an even number of phonons will not depend on the dot, it is insensitive to the state of the electron, and the amplitudes of the electron tunneling from $|+\rangle$ and $|-\rangle$ will still interfere destructively.

In contrast, emission processes involving an odd number of quanta introduce a negative sign for an electron starting in state $|-\rangle$, "detecting" the path (or rather, the initial state) of the electron and interfering constructively with the processes from $|+\rangle$. This lifts the destructive interference and makes $\Gamma \neq 0$ at $\varphi=\pi$. However, below the frequency $\omega$ of the oscillator, destructive interference at $\varphi=\pi$ is still perfect since no quantum can be emitted, while the magnitude of $\Gamma$ at $\varphi$ $=0$ is renormalized by the factor $z$, as has been discussed above in general for the limiting case $V \rightarrow 0$. The same holds true for any bath with a finite excitation gap, at $T=0$. This is shown in Figs. 4 and 5, to be discussed in the following section.

We now pass on to arbitrary bath spectra. At first, we will cover the case $z \neq 0$ ("weak baths"), when we can apply perturbation theory to discuss the behavior of $P_{(-)}(E)$ at low energy transfers $E$ (and, consequently, that of $\Gamma$ at low voltages). A Taylor expansion of the exponent in Eq. (12) yields

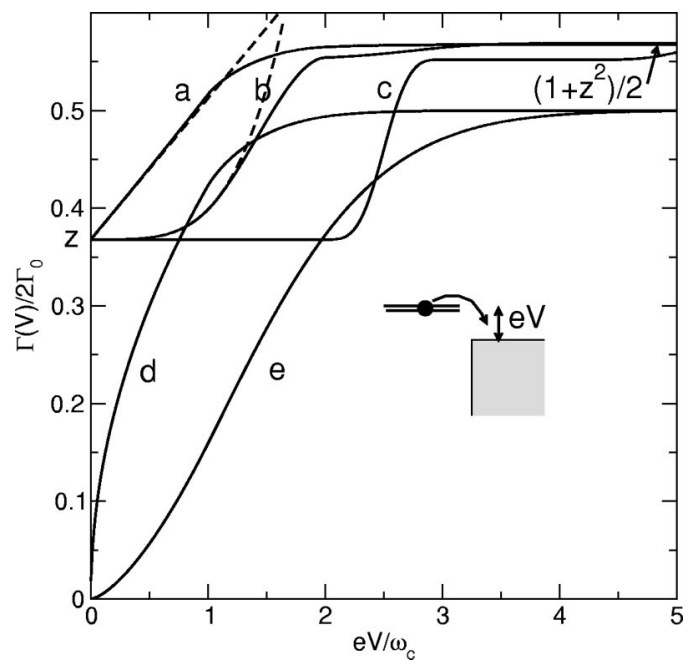

FIG. 4. Decay rate $\Gamma$ as a function of bias voltage $V$ for the case of constructive interference $(\varphi=0)$, at $T=0$. Curves correspond to different bath spectra shown in Fig. 3. Dashed lines correspond to approximation Eq. (28). The initial Coulomb-blockade type suppression to a value of $\Gamma / 2 \Gamma_{0}=z(z=0$ for the Ohmic bath $\mathrm{d}$, e $)$ is lifted with increasing bias voltage, saturating at $\Gamma / 2 \Gamma_{0}=(1$ $\left.+z^{2}\right) / 2$. Inset depicts energy diagram with definition of bias voltage for this situation.

$$
\begin{aligned}
P_{(-)}(E) & =\frac{z}{2 \pi} \sum_{n=0}^{\infty} \frac{1}{n !} \int_{-\infty}^{+\infty} d t e^{i E t}[ \pm\langle\hat{\phi}(t) \hat{\phi}\rangle]^{n} \\
& =z \sum_{n=0}^{\infty} \frac{( \pm 1)^{n}}{n !}\left(\langle\hat{\phi} \hat{\phi}\rangle_{\omega} * \ldots *\langle\hat{\phi} \hat{\phi}\rangle_{\omega}\right)(E) .
\end{aligned}
$$

The repeated convolution product contains $n$ times the correlator $\langle\hat{\phi} \hat{\phi}\rangle_{\omega}$, for $n=0$ it is to equal $\delta(E)$, and the negative sign holds for $P_{-}(E)$.

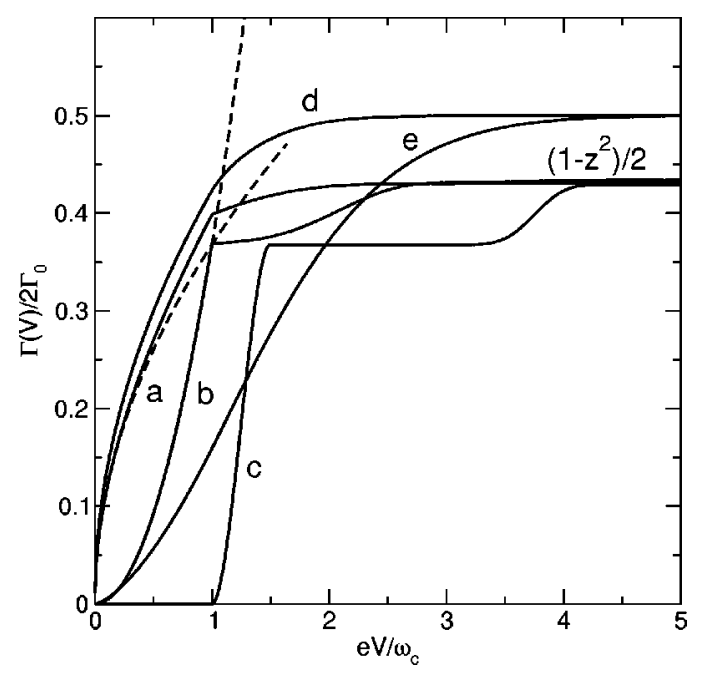

FIG. 5. Decay rate $\Gamma(V)$ for the case of destructive interference ( $\varphi=\pi$ ), at $T=0$. Dashed lines refer to Eq. (28). Due to dephasing, the decay rate becomes finite at finite voltages, saturating at $\Gamma / 2 \Gamma_{0}=\left(1-z^{2}\right) / 2$. For the Ohmic bath $(\mathrm{d}, \mathrm{e})$ the dependence is exactly equal to that for $\varphi=0$ (Fig. 4). 
For the following discussion, we prescribe the spectrum of the fluctuating potential $\hat{F}$ to be a power law in frequency $\omega($ at $T=0)$, with exponent $s$ :

$$
\langle\hat{F} \hat{F}\rangle_{\omega}^{T=0}=2 \alpha \omega_{c}\left(\frac{\omega}{\omega_{c}}\right)^{s} \theta\left(\omega_{c}-\omega\right) \theta(\omega) .
$$

The dimensionless parameter $\alpha$ characterizes the bath strength. In order to be able to rely on perturbation theory, we have to ensure $z>0$. Since $\langle\hat{\phi} \hat{\phi}\rangle_{\omega}=\langle\hat{F} \hat{F}\rangle_{\omega} / \omega^{2}$, the variance of the fluctuating phase, $\left\langle\hat{\phi}^{2}\right\rangle$, will be finite only for $s>1$ (at $T=0$, otherwise $s>2$ ). In that case, we have $z$ $=\exp [-2 \alpha /(s-1)]$. This means the perturbative analysis presented above is restricted to a super-Ohmic bath, $s>1$. The case of the Ohmic bath will be discussed separately further below.

After keeping only terms up to second order in the expansion of $P_{(-)}(E)$ given in Eq. (24), we get

$$
P(E)+P_{-}(E)=z\left[2 \delta(E)+\left(\langle\hat{\phi} \hat{\phi}\rangle_{\omega} *\langle\hat{\phi} \hat{\phi}\rangle_{\omega}\right)(E)+\cdots\right]
$$

for the symmetric combination, which will determine the prefactor of $1+\cos (\varphi)$ in the expression for $\Gamma$, Eq. (13), and

$$
P(E)-P_{-}(E)=2 z\langle\hat{\phi} \hat{\phi}\rangle_{E}+\cdots
$$

for the antisymmetric combination [determining the prefactor of $1-\cos (\varphi)]$. Inserting these into Eq. (13), using the power law for $\langle\hat{\phi} \hat{\phi}\rangle_{\omega}=\langle\hat{F} \hat{F}\rangle_{\omega} / \omega^{2}$ given by Eq. (25), and performing the energy integrals, we find, for sufficiently low voltages $\left[2 \alpha\left(e V / \omega_{c}\right)^{s-1} \ll s-1\right]$,

$$
\begin{aligned}
\Gamma \approx & \frac{\Gamma_{0}}{2} z\left\{[1+\cos (\varphi)]\left[1+\frac{\alpha^{2} C_{s}}{(s-1)}\left(\frac{e V}{\omega_{c}}\right)^{2(s-1)}\right]\right. \\
& \left.+[1-\cos (\varphi)] \frac{2 \alpha}{s-1}\left(\frac{e V}{\omega_{c}}\right)^{s-1}\right\} .
\end{aligned}
$$

The numerical prefactor $C_{s}$ is defined as $\int_{0}^{1}[y(1$ $-y)]^{s-2} d y$.

From Eq. (28), we see that the destructive interference at $\varphi=\pi$ is perfect at $V=0$, but gets lifted when increasing the bias voltage, with a power $V^{s-1}$. In contrast, the decay rate $\Gamma$ at $\varphi=0$ starts out from the constant value of $2 z \Gamma_{0}$ and grows as $V^{2(s-1)}$. Therefore, the visibility $v$ starts out at 1 for $V=0$ but decreases as

$$
v \approx 1-\frac{4 \alpha}{s-1}\left(\frac{e V}{\omega_{c}}\right)^{s-1} .
$$

For $s \downarrow 1$, the range in bias voltage $V$ where these approximate expressions hold shrinks to zero (at constant $\alpha$ and $\left.\omega_{c}\right)$. At $s=1$, i.e., for the Ohmic bath, the probability $z$ of not emitting energy into the bath vanishes completely. As discussed above, this means that there is no $\varphi$ dependence at all in $\Gamma$, and, consequently, the visibility is zero at all bias voltages. Furthermore, the tunneling rate vanishes for $\mathrm{eV}$ $\rightarrow 0$, even at $\varphi=0$. This is the well-known Coulombblockade type of behavior for tunneling in presence of Ohmic dissipation. ${ }^{35}$ At higher bias voltages, the blockade is removed and $\Gamma$ grows towards $\Gamma_{0}$. The growth at low voltages is determined by the power-law behavior of $P(E)$, which rises as $c \omega_{c}^{-2 \alpha} E^{2 \alpha-1}$, where the exponent is determined by the bath strength rather than the exponent $s=1$ of the bath spectrum. The dimensionless prefactor $c$ must be found from the normalization condition for $P(E)$ and depends only on $\alpha$ (and the type of cutoff in the bath spectrum). Therefore, in the case of the Ohmic bath we have, at low $V$ and $T=0$,

$$
\Gamma(V)=\Gamma_{0} \frac{c}{2 \alpha}\left(\frac{e V}{\omega_{c}}\right)^{2 \alpha}
$$
$T>0$.

Finally, we briefly discuss the case of finite temperatures,

In that case, the variance of $\hat{\phi}$ is given by

$$
\left\langle\hat{\phi}^{2}\right\rangle=\int_{0}^{\infty} d \omega\langle\hat{\phi} \hat{\phi}\rangle_{\omega}^{(T=0)} \operatorname{coth}\left(\frac{\omega}{2 T}\right)
$$

which yields

$$
\left\langle\hat{\phi}^{2}\right\rangle \approx\left\langle\hat{\phi}^{2}\right\rangle^{(T=0)}+4 \alpha\left(\frac{T}{\omega_{c}}\right)^{s-1} \int_{0}^{\infty} \frac{y^{s-2}}{e^{y}-1} d y .
$$

The approximation of extending the integral to infinity holds for temperatures much smaller than the bath cutoff $\omega_{c}$. This formula gives the temperature dependence of the renormalization factor $z=\exp \left(-\left\langle\hat{\phi}^{2}\right\rangle\right)$. The second integral diverges for $s \leqslant 2$, because $z=0$ for these cases, in contrast to $T=0$ where $z=0$ only for $s \leqslant 1$. Again, this results in complete absence of the interference effect in the tunneling rate $\Gamma(V, \varphi)$ [because $P_{-}(E)$ vanishes]. It may seem surprising that an infinitesimally small temperature can yield such a drastic qualitative change (for $1<s \leqslant 2$ ), compared to the zero-temperature case, since the difference should be observable only at very large times $t \gg 1 / T$. However, it must be remembered that our analysis is carried out for the limit $\Gamma_{0}$ $\rightarrow 0$, where the average decay time of the given state is inifinitely large. In other words, the limits $T \rightarrow 0$ and $\Gamma_{0}$ $\rightarrow 0$ do not commute for such relatively strong baths. At finite $\Gamma_{0}$, the transition from one to the other case should turn out to be smooth, but this goes beyond the present analysis.

Apart from the change in $z$ with temperature, there are two other important differences to the case $T=0$. First of all, even at $V \rightarrow 0$ the electron may emit energy into the bath, due to the thermal smearing of the Fermi surface in the lead (lifting of Pauli blocking). Second, it may now also absorb some energy during the tunneling process. Both facts will, in general, lead to a finite tunneling decay rate at $\varphi=\pi, V$ $\rightarrow 0$ for any bath, where, at $T=0$, the rate had vanished in any case. 
We can approximate the visibility $v$ at $V \rightarrow 0$ and finite $T$ by using expansion (24). Inserting the resulting expressions for $\gamma_{(-)}$(14) into $v=\gamma_{-} / \gamma$, we obtain

$$
v(T, V \rightarrow 0) \approx 1-4 \int d \epsilon\langle\hat{\phi} \hat{\phi}\rangle_{\epsilon} f(\epsilon) .
$$

We evaluate the integral for a power-law bath spectrum in the limit $T \ll \omega_{c}$,

$$
\begin{aligned}
\int d \epsilon\langle\hat{\phi} \hat{\phi}\rangle_{\epsilon} f(\epsilon) & =\int_{0}^{\infty} d \epsilon \frac{\langle\hat{\phi} \hat{\phi}\rangle_{\epsilon}^{T=0}}{\sinh (\beta \epsilon)} \\
& \approx 2 \alpha \omega_{c}^{1-s} \int_{0}^{\infty} d \epsilon \frac{\epsilon^{s-2}}{\sinh (\beta \epsilon)} .
\end{aligned}
$$

This yields

$$
1-v(T, V \rightarrow 0) \approx 32 \alpha\left(\frac{T}{\omega_{c}}\right)^{s-1}\left(\frac{1}{2}-2^{-s}\right) \Gamma(s-1) \zeta(s-1),
$$

where $\Gamma$ is the Euler gamma function, and $\zeta$ the Riemann zeta function. Therefore, the decrease of the visibility with increasing temperature $T$ (and $V \rightarrow 0$ ) is governed by the same power law as that for increasing bias voltage $V$ at $T$ $=0$, see Eq. (29).

\section{DISCUSSION OF THE RESULTS}

The following discussion relates to the results obtained for $T=0$, which are plotted in the figures.

In Fig. 3, several different types of bath spectra $\langle\hat{F} \hat{F}\rangle_{E}$ are shown. Cases a, b, d, and e are power laws of the form given in Eq. (25), for a cutoff frequency of $\omega_{c}=1$. The last two (d, e) are of Ohmic type $(s=1, z=0)$, which corresponds physically to gate voltage fluctuations due to Nyquist noise. Case $\mathrm{c}$ represents a bath with an excitation gap (for example, optical phonons), with a spectrum given by an inverted parabola. In the limit of infinitely small spectral bandwidth, it would correspond to the single harmonic oscillator (Einstein mode) discussed above. Case $b$, with a bath spectrum rising as $\omega^{3}$, corresponds to the experimentally relevant case of piezoelectric coupling to acoustic phonons, which was determined to be the major inelastic mechanism in the experiments of Ref. 12 on double dots in GaAs (see Ref. 16 for a theoretical analysis deriving this spectrum for wavelengths larger than the dot distance). The spectra for the first three cases $(\mathrm{a}, \mathrm{b}, \mathrm{c})$ have been chosen to give the same renormalization factor, $z=1 / e$. The same figure shows the resulting functions $P(E)$ and $P_{-}(E)$. These have been obtained using the integral equation described in Refs. 32 and 36 . We recall that the low-energy behavior of $P(E)$ is given by $\langle\hat{\phi} \hat{\phi}\rangle_{E}$ $=\langle\hat{F} \hat{F}\rangle_{E} / E^{2}$ for the cases with $z \neq 0$, where perturbation theory may be applied. In case c, the alternating signs of the different contributions to $P_{-}(E)$ may be observed, whose physical meaning has been explained above for the limiting case of the harmonic oscillator.

We now briefly mention some numerical estimates for the bath strengths as they may occur in experimental situations.

In GaAs, the lack of inversion symmetry leads to piezoelectric fields proportional to the lattice deformation, whose effect on electrons at low frequencies is much stronger than that of the usual deformation potential (where it is only the potential that is proportional to the deformation). For the piezoelectric coupling ${ }^{37}$ to acoustic phonons in GaAs, one finds (compare Ref. 16) $\langle\hat{\phi} \hat{\phi}\rangle_{\omega}^{T=0}=W \omega /\left(c_{s} / d\right)^{2}$ for $\omega$ $\ll c_{s} / d$, where $c_{s} \approx 5 \times 10^{3} \mathrm{~m} / \mathrm{s}$ is an estimate for the average velocity of longitudinal sound waves in GaAs, and $d$ denotes the distance between the quantum dots. We obtain $W$ $=\operatorname{const}\left(e h_{14} / 4 \pi\right)^{2} /\left(\hbar \rho c_{s}^{3}\right)$, where $e h_{14}=1.4 \mathrm{eV} / \mathrm{nm}$ is the single piezoelectric modulus in the cubic $T_{d}$ structure of GaAs and $\rho=5.3 \times 10^{3} \mathrm{~kg} / \mathrm{m}^{3}$ the mass density. The numerical constant is of order 1 and accounts for the details of the sound wave dispersion relation as well as the orientation of the crystal axes with respect to the vector separating the quantum dots. Inserting these values, $W$ is found to be on the order of 0.01. In order to obtain the renormalization factor $z$, the spectrum $\langle\hat{\phi} \hat{\phi}\rangle_{\omega}$ must be integrated over all frequencies (see above), i.e., up to the cutoff frequency $\omega_{c}$. The effective cutoff frequency $\omega_{c} \propto c_{s} / d_{0}$ is determined by the extent $d_{0}$ of the dot wave functions (for $d_{0}=100 \mathrm{~nm}$ one obtains $\omega_{c}$ $\sim 50 \mathrm{GHz}$ ). Given the present values, and assuming $d_{0} \approx d$, this leads to estimates for $\int\langle\hat{\phi} \hat{\phi}\rangle_{\omega} d \omega$ on the order of 0.01 , yielding $z=\exp \left(-\left\langle\hat{\phi}^{2}\right\rangle\right)$ near 1 . Note that the distance $d$ between the dots cancels in the estimate for $z$, as long as the cutoff frequency is assumed to be given by $\omega_{c} \propto c_{s} / d$. However, as $\omega_{c}$ might be considerably larger than $c_{s} / d$ (if $d_{0}$ $\ll d$ ), one could also obtain a $z$ that deviates more strongly from unity.

For the Ohmic bath, we may imagine the quantum dots placed inside a capacitor $C$ connected to a circuit of resistance $R$, such that the potential difference $2 \hat{F}$ between the dots would be given by the fluctuating voltage drop across the capacitor. This leads to a bath spectrum $\langle\hat{F} \hat{F}\rangle_{\omega}^{T=0}$ $=\pi\left(R / R_{Q}\right) \hbar^{2} \omega /\left[1+(R C \omega)^{2}\right]$, with $R_{Q}=h / e^{2}$ the quantum of resistance. Therefore, the dimensionless coupling constant $\alpha$ introduced above would be equal to $\alpha=(\pi / 2) R / R_{Q}$, which can have values both larger and smaller than 1 .

Finally, for optical phonons, we use the Fröhlich interaction Hamiltonian (Ref. 34) with a dimensionless Fröhlich coupling constant of $\alpha=0.07$ (GaAs) to obtain the rough estimate $\quad\langle\hat{F} \hat{F}\rangle_{\omega}^{T=0}=\delta\left(\omega-\omega_{L O}\right)(1 \mathrm{meV})^{2}\left(100 \mathrm{~nm} / d_{0}\right)$, with $\omega_{L O} \approx 5 \times 10^{13} \mathrm{~Hz}$. This yields a $z$ deviating from unity by about $10^{-3}$.

However, in the plots we have chosen $z=1 / e$ for illustrative purposes.

The resulting behavior of $\Gamma(\varphi, V)$ at $T=0$, calculated from Eq. (13), is shown in Figs. 4 and 5. In the case of constructive interference $(\varphi=0$, Fig. 4$)$, the decay rate for the "weak baths" (a, b, c) starts out from $\Gamma / 2 \Gamma_{0}=z$ at $V$ $=0$ and goes to $\Gamma / 2 \Gamma_{0}=\left(1+z^{2}\right) / 2$ at $e V / \omega_{c} \gg 1$. The initial deviation from the constant value of $z$ at low voltages is given by the power law $V^{2(s-1)}$ contained in Eq. (28). In contrast, the decay rate for the Ohmic bath $(\mathrm{d}, \mathrm{e})$ starts at $\Gamma=0$, rising with a power law and saturating at a value of 


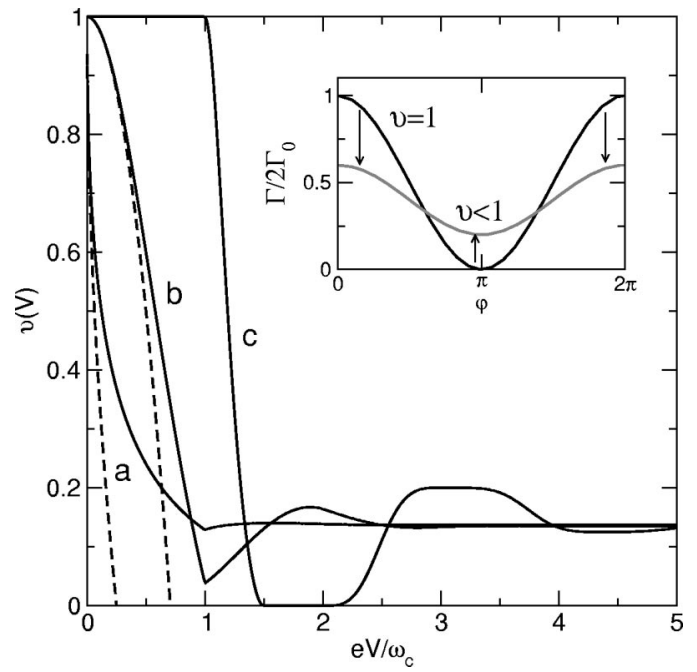

FIG. 6. Visibility $v=\left(\Gamma_{\varphi=0}-\Gamma_{\varphi=\pi}\right) /\left(\Gamma_{\varphi=0}+\Gamma_{\varphi=\pi}\right)$ as a function of bias voltage $V$ for different bath spectra (see Fig. 3). For the Ohmic bath (cases d, e) $v \equiv 0$. Dashed lines correspond to Eq. (29). Inset illustrates change in interference pattern $\Gamma(\varphi)$ upon switching on the interaction with the bath.

$\Gamma / 2 \Gamma_{0}=1 / 2$, corresponding to an equal admixture of odd and even states in the reduced density matrix of the electron coupled to the bath. For destructive interference $(\varphi=\pi$, Fig. 5 ), the behavior of a and $\mathrm{b}$ at low voltages is given by $V^{s-1}$ [see Eq. (28)], while the decay rate of the Ohmic bath (d, e) remains the same as that for $\varphi=0$. In the special case $c$ of the gapped bath, we observe perfect destructive interference up to the excitation threshold of the bath at $e V=\omega_{c}$, where $\Gamma(\varphi=\pi, V)$ increases in a stepwise manner for the first time, with the next increase at $e V=3 \omega_{c}$. Note that, on the other hand, $\Gamma(\varphi=0, V)$ increases at even multiples of the excitation gap. The difference comes about because it is only the emission of an odd number of phonons into the bath that reveals the location of the electron, as discussed above. This feature would be absent if the two dots were coupled to two independent baths, whereas the other qualitative properties would remain the same.

From the decay rates at $\varphi=0$ and $\varphi=\pi$, we may calculate the visibility $v$ of the "interference pattern" that is defined by the dependence of $\Gamma$ on $\varphi$. The result is shown in Fig. 6. As we have noted before, the visibility is always zero for the Ohmic bath. On the other hand, for the "weak baths," it is perfect (equal to 1 ) at $V \rightarrow 0$, due to the perfect destructive interference, regardless of the suppression factor $z$ appearing in $\Gamma(\varphi=0)$. In general, the visibility decreases towards higher bias voltages before saturating at the limiting value of $z^{2}$. However, in contrast to intuitive expectation, the decrease may be nonmonotonous, i.e., the visibility of the interference effect may actually be enhanced by increasing the supply of energy available to the electron, although the decay rate $\Gamma$ always increases monotonously at any $V$. This is particularly striking in case $\mathrm{c}$, where the visibility drops down to zero in a certain range before rising again. The decrease down to the exact value of 0 is related to the special choice of $\left\langle\hat{\phi}^{2}\right\rangle=1 \quad(z=1 / e)$, which gives equal strengths of the peak at $E=0$ and the first peak around $E=\omega_{c}$, which then are able to cancel in the integral $\gamma_{-}$over $P_{-}(E)$ that is proportional to the visibility. However, the physical reason for a dip in visibility is rather generic: In that energy range, the decay rate $\Gamma$ for $\varphi=\pi$ has already increased due to dephasing, while the blockade-type suppression of the value of $\Gamma$ for $\varphi=0$ has not yet been lifted. This is a consequence of the even-odd effect discussed above.

\section{SEQUENTIAL TUNNELING THROUGH THE DOUBLE DOT}

Up to now, we have discussed in detail the influence of the bath on the tunneling decay rate of an electron which has been placed onto the two dots in the symmetric superposition. In order to complete the picture, we have to calculate the sequential tunneling current through such a double-dot interference setup. This will be done by deriving and solving a master equation for the reduced density matrix of the double-dot system, taking into account the system-bath coupling exactly, while the tunnel coupling is treated in leading order. We are interested specifically in the nonlinear response, i.e., in how an increasing bias voltage helps to destroy the phase coherence. The tunneling rates calculated previously will serve as input to the master equation.

However, in order to facilitate the understanding of the results, we first turn to a qualitative description of the situation without the bath.

At $\varphi=\pi$, tunneling is completely blocked, since the left reservoir only couples to the even state $|e\rangle$, while the right reservoir couples to the antisymmetric (odd) superposition, $|o\rangle$. At $\varphi=0$, both reservoirs couple to $|e\rangle$, whereas $|o\rangle$ is completely decoupled from the leads (compare the discussion in Ref. 27). This means that a current may flow if $|o\rangle$ is empty. However, if $|o\rangle$ is filled, the current vanishes, because double occupancy is forbidden in our model. Since there is no way to change the occupation of $|o\rangle$, the stationary density matrix of the double dot at $\varphi=0$ will be any convex combination of these two possibilities (at $T=0$, in the absence of other relaxation paths). At any value of $\varphi$ in between these extremes, there is always the state $|\Psi\rangle=(|+\rangle$ $\left.-e^{-i \varphi}|-\rangle\right) / \sqrt{2}$, whose decay into the right lead is blocked by destructive interference. As there is a nonvanishing overlap between $|\Psi\rangle$ and the state $|e\rangle$ which is reached by tunneling from the left lead, one will observe an accumulation of population in $|\Psi\rangle$, until the current is blocked again. This argument holds at $T=0$, while at finite temperatures the electron can decay towards the left lead and make a new attempt. Therefore, in this simple picture, the stationary current at $T=0$ would be zero at any $\varphi$ except for $\varphi=0$, where it is undefined.

However, one has to take into account that the coupling to the reservoirs does not only lead to decay but also to an effective tunnel coupling between $|+\rangle$ and $|-\rangle$. Although this cannot change the blockade of the current at $\varphi=\pi$ (leading only to an energy shift of $|e\rangle$ vs $|o\rangle)$, it does lift the blockade at other values of $\varphi$. This is because the blocked state $|\Psi\rangle$ is no longer stationary, such that an electron will not remain there forever. The degeneracy at $\varphi=0$ still remains. Therefore, in the ideal case without coupling to a 
bath, we expect the current to vanish at $\varphi=\pi$ and to rise towards a maximal amplitude near $\varphi=0$. According to the previous argument, at $T=0$ this maximal amplitude will be determined by the effective tunnel coupling between the dot states.

Introducing the bath will then lead to renormalization effects and spoil the perfect destructive interference at higher values of the bias voltage (or temperature), qualitatively in the same way as has been explained above. We will show that the actual visibility $v_{I}$ of the current interference pattern $I(\varphi)$ is given by a monotonous function of the visibility $v$ introduced above for the tunneling rate (at symmetric bias).

We start with the Hamiltonian that is obtained after applying the unitary transformation of the independent boson model (A5) onto our Hamiltonian (1),

$$
\hat{H}^{\prime}=\epsilon^{\prime}\left(\hat{n}_{+}+\hat{n}_{-}\right)+U^{\prime} \hat{n}_{+} \hat{n}_{-}+\hat{H}_{B}+\hat{H}_{L}+\hat{H}_{R}+\hat{V}^{\prime} .
$$

Here $\epsilon^{\prime}$ is the (renormalized) energy of the two states, which we will take to be $\epsilon^{\prime}=0$ from now on. $U^{\prime}$ is the interaction constant that involves both the Coulomb repulsion as well as the effective attractive interaction induced by the bath. We assume $U, U^{\prime} \gg T, e V$, such that double occupancy is forbidden.

The term which we will treat as a perturbation is given by $\hat{V}^{\prime}$, describing the tunneling to the left and the right leads in the presence of the bath. It is the transformed version of $\hat{V}$ [compare Eqs. (3) and (4) and Appendix A], where the additional fluctuating phase factors $\exp ( \pm i \hat{\phi})$ have been introduced:

$$
\hat{V}^{\prime}=\sum_{j=l, r} \sum_{\alpha=+,-} \hat{j}_{\alpha} \hat{d}_{\alpha}+\text { H.c. }
$$

where

$$
\begin{gathered}
\hat{l}_{ \pm}=e^{ \pm i \hat{\phi}} \hat{l}, \\
\hat{l}=\sum_{k} t_{k}^{L} \hat{a}_{L k}^{\dagger}, \\
\hat{r}_{+}=e^{+i \hat{\phi}} \hat{r}, \\
\hat{r}_{-}=e^{-i \hat{\phi}} e^{i \varphi} \hat{r}, \\
\hat{r}=\sum_{k} t_{k}^{R} \hat{a}_{R k}^{\dagger} .
\end{gathered}
$$

As usual, the current through the device does not only depend on the rates for electrons to tunnel into and out of the dots, but also on the stationary state which the system assumes in the nonequilibrium situation, i.e., under an applied bias voltage.

We will now derive a master equation for the reduced density matrix $\hat{\rho}$ of the double-dot system, which contains the populations $\rho_{++}, \rho_{--}, \rho_{00}$ (" 0 " denoting "no electron") and the coherences $\rho_{+-}$and $\rho_{-+}$(with $\rho_{00}=1$ $-\rho_{++}-\rho_{--}, \rho_{\alpha 0}=\rho_{0 \alpha}=0$ for $\alpha \neq 0$, and $\rho_{-+}=\rho_{+-}^{*}$ ). We cannot simply use the standard kind of master equation, since we have to deal with two degenerate levels $|+\rangle$ and $|-\rangle$, and it is important that a tunneling event may create a coherent superposition of $|+\rangle$ and $|-\rangle$ (for example, the even state $|e\rangle)$. Such a master equation-for degenerate levels-has also been employed in Ref. 11 (without coupling to the bath, and evaluated in the linear-response regime). The equation is different from that employed in the "orthodox" theory of sequential tunneling, where no coherent superpositions are involved. Note that for a finite tunnel coupling the levels could be treated as degenerate as long as their energetic distance is much smaller than the level broadening due to tunneling. However, as we consider the limit $\Gamma_{0} \rightarrow 0$, we need to have exactly equal energies. Otherwise, the energy of the hole that is created in the left electrode would betray the dot state which the electron has entered, thus preventing any coherent superposition to form.

Given the initial reduced density matrix $\hat{\rho}(0)$, and assuming the state of the environment (bath and reservoirs) to be independent of the electronic state on the dot at $t=0$, we obtain the time evolution $\hat{\rho}(t)$ by tracing over the environmental degrees of freedom (" $E$ "),

$$
\begin{aligned}
\hat{\rho}(t)= & \operatorname{tr}_{E}\left[\hat{T} \exp \left(-i \int_{0}^{t} d s \hat{V}^{\prime}(s)\right) \hat{\rho}(0) \otimes \hat{\rho}_{E}\right. \\
& \left.\times \widetilde{\hat{T}} \exp \left(i \int_{0}^{t} d s \hat{V}^{\prime}(s)\right)\right] \\
= & \hat{\rho}(0)-\int_{0}^{t} d t_{1} \int_{0}^{t_{1}} d t_{2} \operatorname{tr}_{E}\left[\hat{V}^{\prime}\left(t_{1}\right) \hat{V}^{\prime}\left(t_{2}\right)\right. \\
& \left.\times \hat{\rho}(0) \otimes \hat{\rho}_{E}+\text { H.c. }\right]+\int_{0}^{t} d t_{1} \int_{0}^{t} d t_{2} \\
& \times t_{E}\left[\hat{V}^{\prime}\left(t_{1}\right) \hat{\rho}(0) \otimes \hat{\rho}_{E} \hat{V}^{\prime}\left(t_{2}\right)\right]+\cdots
\end{aligned}
$$

Physically, by using factorized initial conditions, we neglect correlations between subsequent tunneling events which could be due to excitations in the electrodes or in the bath. Since the tunneling rate is very small, these excitations will have traveled away from the double dot until the next event takes place. The entanglement between electron and bath (discussed in the previous sections) would preclude factorized initial conditions, if it were not treated indirectly in this approach (via the unitary transformation). Note that we do not have to make any secular approximation at this point, unlike the usual derivation of a master equation. ${ }^{38}$ It turns out that all contributions only depend on the time difference $t_{1}-t_{2}$ anyway, because the dot levels are degenerate. Therefore, in the long-time limit $t \rightarrow \infty$, the integration over $\left(t_{1}\right.$ $\left.+t_{2}\right) / 2$ results in a factor $t$, and the endpoints of the integrals over $t_{1}-t_{2}$ may be extended to $\infty$. This yields the desired master equation that will determine the stationary $\hat{\rho}$, as well as the current, in the limit of weak tunnel coupling. Some details of the derivation are provided in Appendix B. We also point out that it is possible to handle even the case of non- 
degenerate levels correctly (at least for the case without the bath), by omitting the secular approximation from the outset. ${ }^{39}$

In order to abbreviate the resulting expressions, we introduce the following definitions for the effective in- and outtunneling rates as well as the effective tunnel couplings generated by the electrodes:

$$
\begin{gathered}
\gamma_{L(-)} \equiv \Gamma_{L 0} \int d \epsilon\left[1-f_{L}(\epsilon)\right] P_{(-)}(-\epsilon), \\
\gamma_{L(-)}^{i n} \equiv \Gamma_{L 0} \int d \epsilon f_{L}(\epsilon) P_{(-)}(\epsilon), \\
\Delta_{L} \equiv-\frac{\Gamma_{L 0}}{\pi} \int_{-\infty}^{\Lambda} d \epsilon\left[1-f_{L}(\epsilon)\right] \int d \omega \frac{P_{-}(\omega)}{\epsilon+\omega}, \\
\tilde{\gamma}_{L_{-}} \equiv \gamma_{L-}[P \mapsto \widetilde{P}]=\gamma_{L_{-}}+i \Delta_{L} .
\end{gathered}
$$

Analogous definitions hold for $L \mapsto R$.

Equation (44) is equivalent to definition (14) used for $\gamma_{(-)}$in previous sections. Note that the effective tunnel coupling $\Delta_{L(R)}$ depends on $P_{-}$, because it arises from transitions between the states $|+\rangle$ and $|-\rangle$, via an intermediate lead state. In the expression for $\Delta_{L(R)}$, the energy dependence of the density of states and the tunnel coupling to the reservoir electrode should be kept in order to have a convergent integral. We will take this into account by introducing an effective upper energy cutoff $\Lambda$ in the integral.

One might wonder why the effective tunnel couplings $\Delta_{L(R)}$ depend on the occupation of electron states in the reservoirs. After all, in the noninteracting case, it is possible to calculate such a change of the effective single-particle Hamiltonian prior to filling in the electron states. Alternatively, in a calculation that already takes into account occupation factors, there would be two contributions which add up to an integral that does not depend on the Fermi function. However, we consider the interacting case $U=\infty$, such that (even without the bath) one of these contributions is missing (since it would involve intermediate states with double occupancy). The resulting logarithm is analogous to that which appears in the Kondo problem. This effective tunnel coupling has also been discussed in Ref. 23, for the case without a bath. There, the upper cutoff $\Lambda$ was provided by the Coulomb coupling $U$, since for higher energies double occupancy is no longer forbidden and the noninteracting case takes over (where two contributions arise that cancel each other). If we take the limit $U \rightarrow \infty$, then $\Lambda$ will be set by a cutoff in the tunnel matrix elements (or the electron reservoir's density of states).

The general master equation for the reduced density matrix of the double dot, derived in the limit of weak tunnel coupling but arbitrary electron-bath coupling, follows by inserting definitions (44)-(47) into Eqs. (B2) and (B3):

$$
\begin{array}{r}
\dot{\rho}_{++}=-\rho_{++}\left(\gamma_{L}+\gamma_{R}\right)+\rho_{00}\left(\gamma_{L}^{i n}+\gamma_{R}^{i n}\right) \\
-\frac{\rho_{-+}}{2}\left(e^{i \varphi} \tilde{\gamma}_{R-}+\tilde{\gamma}_{L-}\right)-\text { H.c. }
\end{array}
$$

$$
\begin{gathered}
\dot{\rho}_{--}=-\rho_{--}\left(\gamma_{L}+\gamma_{R}\right)+\rho_{00}\left(\gamma_{L}^{i n}+\gamma_{R}^{i n}\right) \\
-\frac{\rho_{+-}}{2}\left(e^{-i \varphi} \tilde{\gamma}_{R-}+\tilde{\gamma}_{L-}\right)-\text { H.c. } \\
\dot{\rho}_{+-}=-\rho_{+-}\left(\gamma_{L}+\gamma_{R}\right)+\rho_{00}\left(e^{i \varphi} \gamma_{R-}^{i n}+\gamma_{L-}^{i n}\right) \\
-\frac{\rho_{++}}{2}\left(e^{i \varphi} \tilde{\gamma}_{R-}^{*}+\tilde{\gamma}_{L-}^{*}\right)-\frac{\rho_{--}}{2}\left(e^{i \varphi} \tilde{\gamma}_{R-}+\tilde{\gamma}_{L-}\right)
\end{gathered}
$$

The ingredients of the master equation obtained here may be interpreted as follows:

One part of the right-hand side corresponds to the unitary time evolution generated by the effective tunneling Hamiltonian,

$$
\hat{H}_{e f f}^{T}=\frac{1}{2}\left(e^{i \varphi} \Delta_{R}+\Delta_{L}\right)|+\rangle\langle-|+\text { H.c. }
$$

Furthermore, the in-tunneling contributions in the equations for $\rho_{++}$and $\rho_{--}$depend on $P(E)$, while that for $\rho_{+-}$ is determined by $P_{-}(E)$, since it describes the creation of a coherent superposition of $|+\rangle$ and $|-\rangle$ (which is hindered by the bath). This term would be absent in the usual master equation. In particular, if $\gamma_{L-}^{i n} \rightarrow \gamma_{L}^{i n}$, which will be the case at $T=0$ for vanishing bias between the dots and the left electrode, an electron tunneling from the left lead will end up in the coherent superposition where $\rho_{+-}=\rho_{++}=\rho_{--}$. Taking into account that we are working in a transformed basis, this describes just the entangled state (7), confirming the starting point of our earlier discussion. Note that the outtunneling contribution for $\rho_{++}$also depends on $\rho_{+-}$, for example. This reflects the fact that a superposition between the two states may be blocked from decaying into the lead, while each state can decay separately.

The stationary density matrix is obtained by demanding $d \hat{\rho} / d t=0$ (and using the relations $\rho_{00}=1-\rho_{++}-\rho_{--}$and $\rho_{-+}=\rho_{+-}^{*}$ ). This will give us the density matrix in zeroth order $\Gamma_{0}^{0}$ in the bare tunnel coupling, which we need to calculate the current in leading order $\Gamma_{0}^{1}$.

We can obtain the current from the contribution of the left electrode to the change $\dot{\rho}_{++}+\dot{\rho}_{--}$in the double-dot occupation (i.e., keeping only terms that stem from the left electrode in the master equation). This is equal to the right-going current in the stationary limit:

$$
\begin{aligned}
& \frac{I}{e}=\left(\dot{\rho}_{++}+\dot{\rho}_{--}\right)_{L}=2 \rho_{00} \gamma_{L}^{i n}-\gamma_{L}\left(\rho_{++}+\rho_{--}\right) \\
& -2 \gamma_{L-} \operatorname{Re}\left[\rho_{+-}\right]
\end{aligned}
$$

An alternative way of deriving the current would be to start from the general Meir-Wingreen formula ${ }^{40}$ which expresses the current in terms of the exact Green's functions of the double dot, to be calculated in presence of the tunnelcoupling and the bath. This has been the approach of Ref. 11 for the case without the bath, and we have checked Eq. (52) to give the same result in that case. 


\section{EVALUATION OF THE SEQUENTIAL TUNNELING CURRENT AND THE VISIBILITY}

In order to evaluate the current as a function of temperature $T$, bias voltage $V$, and phase difference $\varphi$, we will now specialize to the case of symmetric bias and left-right symmetric tunnel couplings $\left(\Gamma_{R 0}=\Gamma_{L 0}=\Gamma_{0}\right)$. All essential features (in particular, the perfect destructive interference in absence of the bath) are independent of this assumption. We will find that the current is symmetric under $\varphi \mapsto-\varphi$ even for the nonlinear response considered here, due to the symmetry of the model (compare Ref. 11 for a systematic analysis of phase locking in a variety of interference geometries).

We find from Eqs. (44)-(47), using $f(\epsilon)=1-f(-\epsilon)$,

$$
\gamma_{R(-)}=\gamma_{L(-)}^{i n}=\gamma_{(-)} \equiv \Gamma_{0} \int d \epsilon f(\epsilon-\mu) P_{(-)}(\epsilon)
$$

where $\mu=e V / 2$ is the chemical potential of the left reservoir. This is definition (14), with $e V$ replaced by $\mu=e V / 2$ (since we deal with the symmetric bias case). Furthermore, we use the condition of detailed balance, $P_{(-)}(-E)$ $=\exp (-\beta E) P_{(-)}(E)$ (see, for example, Ref. 32), which leads to

$$
\gamma_{L(-)}=\gamma_{R(-)}^{i n}=e^{-\beta \mu} \gamma_{(-)}
$$

The effective tunnel couplings are still different (because of the different Fermi distributions),

$$
\Delta_{L(R)}=-\frac{\Gamma_{0}}{\pi} \int_{-\infty}^{\Lambda} d \epsilon f[-(\epsilon \mp \mu)] \int d \omega \frac{P_{-}(\omega)}{\epsilon+\omega} .
$$

The lower sign belongs to the right electrode.

For the special case of $T=0$, electrons always enter from the left and go to the right, such that we have $\gamma_{L}=\gamma_{L-}$ $=\gamma_{R}^{i n}=\gamma_{R-}^{i n}=0$ and $\gamma_{R(-)}=\gamma_{L(-)}^{i n}=\gamma_{(-)}$, with

$$
\gamma_{(-)}=\Gamma_{0} \int_{0}^{\mu} d \epsilon P_{(-)}(\epsilon) \text {. }
$$

The effective tunnel couplings are, at $T=0$,

$$
\Delta_{L(R)}=-\frac{\Gamma_{0}}{\pi} \int d \omega P_{-}(\omega) \ln \left[\frac{\Lambda+\omega}{|\mu \pm \omega|}\right] \text {. }
$$

Note that, without any bath present, $\Delta_{L(R)}$ will have a logarithmic singularity at $\mu \rightarrow 0$, for $T=0$. The upper cutoff $\Lambda$ will be given by the minimum of the Coulomb repulsion energy $U$ and the bandwidth of the reservoir's electronic energy band (or by some cutoff in the tunnel matrix elements). For the purposes of our discussion, we assume $\Lambda \gg \mu, \omega$.

In the limit of high bias voltages $(\omega \ll \Lambda, \mu)$, we obtain effective tunnel couplings whose magnitude goes as $z^{2}$ and decreases logarithmically with increasing $\mu$,

$$
\Delta_{L} \approx \Delta_{R} \approx-\frac{\Gamma_{0}}{\pi} \ln \left[\frac{\Lambda}{\mu}\right] \int d \omega P_{-}(\omega)=-z^{2} \frac{\Gamma_{0}}{\pi} \ln \left[\frac{\Lambda}{\mu}\right]
$$

By solving the master equation for the stationary density matrix and inserting the result into Eq. (52), we obtain the expression for the current through the double dot in terms of all of the quantities mentioned previously. In general (at arbitrary $T$ ), it is found that the current may be written as the product of $\gamma$ with a dimensionless function of the phase difference $\varphi$ and the ratios $v=\gamma_{-} / \gamma, \delta_{L(R)}=\Delta_{L(R)} / \gamma$, and $\beta \mu$,

$$
I=e \gamma I_{0}\left[\varphi, \beta \mu, v, \delta_{L}, \delta_{R}\right]
$$

The complete expression for $I_{0}$ is very cumbersome, although it may be found analytically by straightforward solution of the master equation (it is listed for $T=0$ in Appendix C). Therefore, let us first discuss the situation without coupling to a bath. In that case, we obtain

$$
\delta_{L}=\delta_{R} \equiv \delta=-\frac{\Gamma_{0}}{\pi} \int_{-\infty}^{\Lambda} \frac{d \epsilon}{\epsilon} f(\mu-\epsilon)
$$

and $\gamma=\gamma_{-}=\Gamma_{0} f(-\mu)$. The current turns out to be (with $\left.\lambda \equiv e^{-\beta \mu}\right)$

$$
\frac{I}{e \gamma}=\frac{4(1-\lambda)\left(\delta^{2}+\lambda\right) \cos ^{2}\left(\frac{\varphi}{2}\right)}{3 \delta^{2}+2\left(1+\lambda+\lambda^{2}\right)+3 \delta^{2} \cos (\varphi)}
$$

Several points should be noticed about this expression. First, the destructive interference at $\varphi=\pi$ remains perfect regardless of temperature, because there are no currentcarrying states at all. At zero temperature $(\lambda=0)$, the maximal amplitude of the current is $I_{\text {max }} / e \gamma=2 \delta^{2} /\left(3 \delta^{2}+1\right)$, which vanishes when the effective tunnel coupling $\delta$ goes to zero. This has been explained above as a consequence of the possible transition into a current-blocking state, which can only be undone by the effective tunnel coupling. At finite temperatures $(\lambda>0)$, the maximal current is nonzero even for $\delta \rightarrow 0$, where it approaches the value of $I_{\text {max }} / e \gamma$ $=2 \lambda(1-\lambda) /\left(1+\lambda+\lambda^{2}\right)$. This has a maximum at around $T \sim \mu$. It vanishes for larger temperatures as $\mu / T$, which is to be expected for tunneling through a localized level (decreasing derivative of the Fermi function). In addition, the shape of $I(\varphi)$ depends on $\delta$ and $\lambda$, with a sharper minimum at $\varphi=\pi$ in the case of larger $|\delta|$. In the limit of $\delta \rightarrow 0$, the current becomes a pure cosine. At finite temperatures (as well as for $v \neq 1$ ) the behavior is similar, except for the finite amplitude of the current at $\delta \rightarrow 0$.

Now we turn to the situation including the bath. The general expression for the current is very lengthy, and we will omit it here. However, it turns out that the maximal and minimal current are functions merely of $v$ and $\lambda=e^{-\beta \mu}$, while they are independent of $\delta_{L, R}$.

The amplitude of the minimal current $($ at $\varphi=\pi)$ is given by

$$
\frac{I(\varphi=\pi)}{e \gamma}=\frac{2(1+\lambda)\left(1-\lambda^{2}\right)\left(1-v^{2}\right)}{3(1+\lambda)^{2}+(1-\lambda)^{2} v^{2}},
$$

while the maximal current (at $\varphi=0$ ) is 


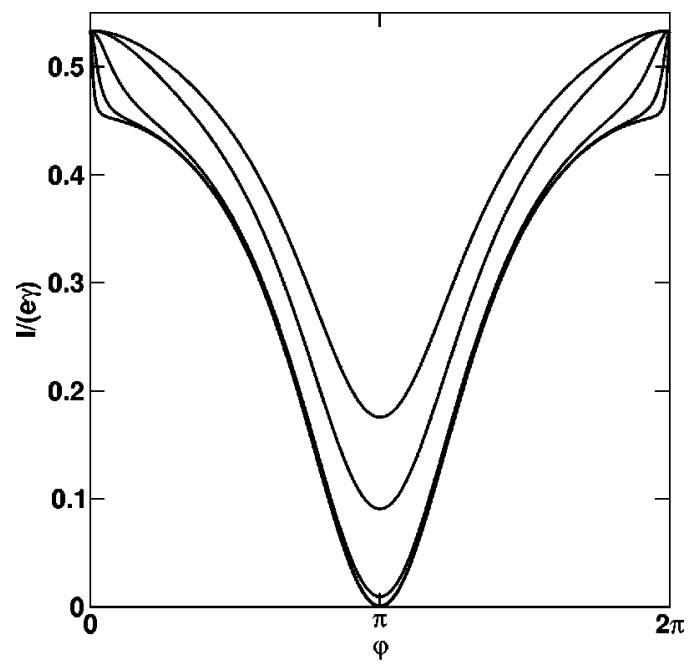

FIG. 7. The current $I$ for different values of the visibility $v$ $=\gamma_{-} / \gamma=0.8,0.9,0.99,0.999,0.9999$ (from top to bottom). The limits $\varphi \rightarrow 0$ and $v \rightarrow 1$ do not commute. Other parameters held fixed: $\lambda=e^{-\beta \mu}=0.2$ and $\delta_{L}=\delta_{R}=-1$.

$$
\frac{I(\varphi=0)}{e \gamma}=\frac{2}{3}(1-\lambda) .
$$

It should be noted that expression (61) for the current in the ideal case seems to contradict this simple formula. However, that is because the limits $\varphi \rightarrow 0$ and $v \rightarrow 1$ do not commute. This is shown in Fig. 7. It means that for $T=0$ and $\delta_{L, R}$ $\rightarrow 0$ the maximal current calculated according to Eq. (63), which is independent of $\delta_{L, R}$, and the "typical" amplitude of the current $\left(\propto \delta_{L}^{2}\right)$ may deviate strongly. The peculiar behavior near $\varphi=0$ seems to be connected to the physical degeneracy of the case $\varphi=0, v=1$ which has been discussed above.

From these formulas, we obtain the visibility, defined in terms of the current,

$$
v_{I} \equiv \frac{I(\varphi=0)-I(\varphi=\pi)}{I(\varphi=0)+I(\varphi=\pi)} .
$$

It can be expressed entirely by the visibility $v$ defined previously in terms of the tunneling rates [Eqs. (16) and (17)], as well as the temperature-dependent factor $\lambda=e^{-\beta \mu}$ $(\mu=e V / 2)$,

$$
v_{I}=\frac{2\left(1+\lambda+\lambda^{2}\right) v^{2}}{3(1+\lambda)^{2}-\left(1+4 \lambda+\lambda^{2}\right) v^{2}} .
$$

This is a monotonous mapping of $v$ to the interval $[0,1]$, with only a weak dependence on $\lambda$. The other parameters $\delta_{L}, \delta_{R}$ only modify the amplitude and shape of the current pattern $I(\varphi)$. Therefore, all the statements about the visibility made in the previous discussion of the tunneling decay out of the symmetric superposition continue to hold up to this monotonous transformation (and with $e V$ replaced by $\mu=e V / 2$ ). In particular, at $T=0$, we have

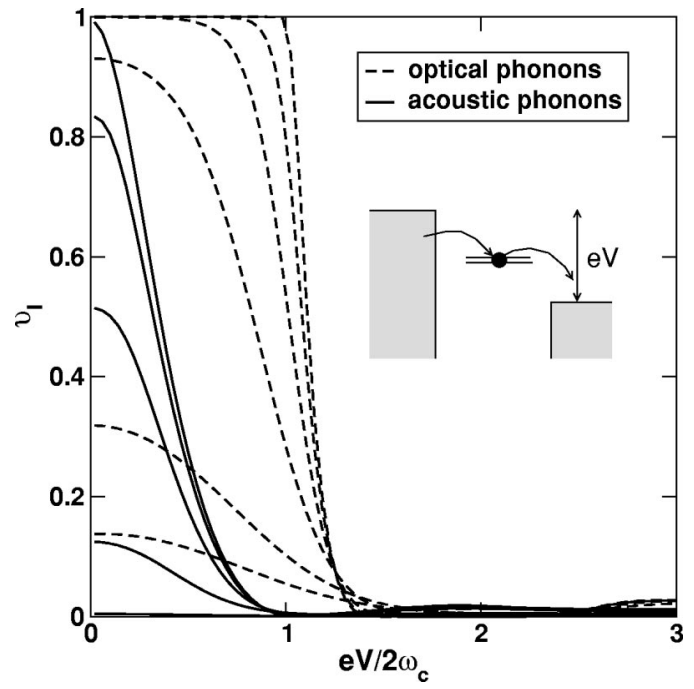

FIG. 8. The visibility $v_{I}$ of the pattern $I(\varphi)$, for piezoelectric coupling to acoustic phonons (b) (solid line) and for the optical phonon bath (c) (dashed line), plotted vs $\mu=e V / 2$, at different temperatures $T / \omega_{c}=0.01,0.05,0.1,0.2,0.4,0.5$ (top to bottom). Inset depicts energy diagram for tunneling in this situation.

$$
v_{I}=\frac{2 v^{2}}{3-v^{2}} .
$$

The dependence of the visibility $v_{I}$ on the bias voltage $e V=2 \mu$, the temperature $T$, and the bath spectrum is displayed in Fig. 8, for bath spectra of type b and c. The decrease of $v_{I}$ at $\mu=0$ with increasing temperature $T$ in case $\mathrm{b}$ is well approximated by Eq. (35) for $v(T, V \rightarrow 0)$ [employing the relation $v_{I}=v^{2} /\left(2-v^{2}\right)$ for $\mu=0$ ]. [The functions $P_{(-)}(E)$ for finite temperatures have been calculated numerically using the fast Fourier transform, from the defining Eq. (12)].

Note that for bath spectra with $z=0$ (i.e., exponent $s \leqslant 1$ at $T=0$ and $s \leqslant 2$ at $T>0$ ) the visibility vanishes entirely (at any $V$ ), as has been explained in the previous sections. We have already pointed out that this picture is expected to change if one treats the tunnel coupling to higher order. However, we have to leave this analysis for the future. One possible approach to a nonperturbative (but still approximate) treatment of both the tunnel-coupling and the systembath coupling at the same time seems to be the numerical "real-time renormalization group" scheme. ${ }^{41}$

\section{CONCLUSIONS}

We have analyzed dephasing in tunneling through two parallel single-level quantum dots with a fluctuating energy difference between the dots. The disappearance of perfect destructive interference in a symmetric setup has been taken as a criterion for "genuine" dephasing, as opposed to mere renormalization. The coupling to the bath has been taken into account exactly, via the "independent boson model" and the concepts of the " $P(E)$ theory" of tunneling in a dissipative environment, while the tunnel coupling has been treated in leading order. 
We have discussed in detail the behavior of the density matrix of a single electron that has been placed in a superposition of the two dot levels. The bath measures (to some extent) the position of the electron, such that the electron's density matrix becomes mixed. However, this allows direct conclusions about the "incoherent current" only in the limit of high bias voltages, corresponding to a fast "projection" measurement of the electron's state. For lower voltages, only the low-frequency part of the bath spectrum contributes to the lifting of destructive interference. Thus, for any "weak bath," whose spectrum falls off fast towards low frequencies, the visibility of the interference effect becomes perfect in the limit of low bias voltages $V$ and temperatures $T$, when the energy supplied to the electron is vanishingly small. This is the case for a fluctuation spectrum $\propto \omega^{s}$ with $s>1(s>2)$ for $T=0 \quad(T>0)$. The visibility may show a nonmonotonous behavior as a function of bias voltage. For "stronger" spectra (smaller exponent $s)$, including the Ohmic bath $(s=1)$, there is the well-known zero-bias anomaly (suppression of the tunneling current at low voltages), which affects equally both the cases of constructive and destructive interference. Therefore, the visibility vanishes exactly at any bias voltage in our approach, where the tunnel coupling has been treated only in leading order. Although there is always a suppression of the magnitude of the tunnel current for the case of constructive interference, this may be interpreted as a mere renormalization of the effective tunnel coupling, since the perfect destructive interference is not affected and since it occurs even for a bath with an excitation gap. The full dependence of the sequential tunneling current $I(\varphi)$ on voltage, temperature, bath spectrum, and phase difference $\varphi$ between the interfering paths has been derived by setting up a master equation for the state of the double dot (which is special due to the degeneracy of dot levels).

The major questions that have remained open in our analysis are related to the behavior at stronger tunnel coupling. In particular, the perfect destructive interference may also be overcome by correlated tunneling of several particles (with an intermediate "virtual" excitation of the bath), and this process will therefore contribute to dephasing, although it is expected to be suppressed strongly at low voltages and temperatures. Likewise, the visibility for the Ohmic bath (or other strong baths), which turns out to be zero in the present approximation, may be changed at low bias voltages and temperatures comparable to the tunneling rate. This will require other methods to analyze the competition between strong tunnel coupling and system-bath coupling.

\section{ACKNOWLEDGMENTS}

We thank J. König, H. Schoeller, H.-A. Engel, D. Loss, and $\mathrm{H}$. Grabert for useful discussions. This work was supported through the Swiss NSF and the Swiss NCCR for Nanoscience.

\section{APPENDIX A: INDEPENDENT BOSON MODEL}

For reference purposes, we describe here the canonical transformation employed in the independent boson model.
See Ref. 34 for more details (concerning the case of at most a single particle). Consider a set of electronic levels $j$ that couple to bath operators $\hat{F}_{j}$ which are assumed to be linear in the coordinates (and momenta) of a bath of harmonic oscillators, $\hat{H}_{B}$,

$$
\hat{H}=\sum_{j}\left(\varepsilon_{j}+\hat{F}_{j}\right) \hat{n}_{j}+\hat{H}_{B} .
$$

Here $\varepsilon_{j}$ is the unperturbed level energy and $\hat{n}_{j}=\hat{d}_{j}^{\dagger} \hat{d}_{j}$ is the number of particles on level $j$. The fluctuating fields are characterized completely by their power spectra at $T=0$,

$$
\left\langle\hat{F}_{l} \hat{F}_{j}\right\rangle_{\omega}^{T=0} \equiv \frac{1}{2 \pi} \int_{-\infty}^{+\infty} d t e^{i \omega t}\left\langle\hat{F}_{l}(t) \hat{F}_{j}\right\rangle^{T=0} .
$$

Here we will restrict ourselves to the case where the different variables commute, $\left[\hat{F}_{l}, \hat{F}_{j}\right]=0$. As a consequence, the spectrum $\left\langle\hat{F}_{l} \hat{F}_{j}\right\rangle_{\omega}^{T=0}$ is real valued, but there may still be correlations.

The most straightforward solution proceeds via a unitary transformation $^{34}$ (essentially a gauge transformation). One introduces the fluctuating phases $\hat{\phi}_{j}$, whose time derivatives are given by the $\hat{F}_{j}$,

$$
\hat{\phi}_{j} \equiv i\left[\hat{H}_{B}, \hat{\phi}_{j}\right]=-\hat{F}_{j} .
$$

The exponent generating the unitary transformation is defined as

$$
\hat{\chi}=\sum_{j} \hat{\phi}_{j} \hat{n}_{j} .
$$

Applying the transformation to the Hamiltonian in Eq. (A1) yields

$$
\hat{H}^{\prime}=e^{-i \hat{\chi}} \hat{H} e^{+i \hat{\chi}}=\sum_{j} \varepsilon_{j} \hat{n}_{j}-\sum_{l j} J_{l j} \hat{n}_{l} \hat{n}_{j}+\hat{H}_{B} .
$$

The coupling between system and bath has been eliminated, resulting in an effective interaction between particles on the different levels, with

$$
J_{l j}=\int_{0}^{\infty} d \omega \frac{\left\langle\hat{F}_{l} \hat{F}_{j}\right\rangle_{\omega}^{T=0}}{\omega} .
$$

The $J_{l j}$ are real valued and independent of temperature. For $l=j$ they describe energy shifts of single-particle levels. The canonical transformation also changes the particle annihilation and creation operators,

$$
\hat{d}_{j}^{\prime}=e^{-i \hat{\chi}} \hat{d}_{j} e^{+i \hat{\chi}}=e^{i \hat{\phi}_{j}} \hat{d}_{j},
$$

and $\hat{d}_{j}^{\prime \dagger}=\hat{d}_{j}^{\dagger} e^{-i \hat{\phi}_{j}}$. This will affect all Green's functions and, therefore, also the time evolution of the single-particle density matrix. In addition, it becomes important if a tunneling part is added to the Hamiltonian, where the operators $\hat{d}_{j}^{(\dagger)}$ appear, such that they have to be transformed according to 
Eq. (A7). However, since the phases $\hat{\phi}_{j}$ and the particle operators $\hat{d}_{j}^{(\dagger)}$ commute (even at different times, when evolved according to $\hat{H}^{\prime}$ ), the evaluation of Green's functions always splits into a part referring to the particles and a separate average over the bath operators. This is the major simplification brought about by the "diagonal coupling" between system and bath.

\section{APPENDIX B: DETAILS OF THE MASTER EQUATION DERIVATION}

The general expression for the time evolution of the reduced density matrix, Eq. (43), may be evaluated by noting that in the expectation values of products $\hat{V}^{\prime} \hat{V}^{\prime}$ only those contributions remain which combine $\hat{d}_{\alpha} \hat{j}_{\alpha}$ (tunneling out of the dots) with $\hat{j}_{\beta}^{\dagger} \hat{d}_{\beta}^{\dagger}$ (tunneling onto the dots),

$$
\begin{aligned}
\frac{d \hat{\rho}}{d t}= & -\sum_{\alpha, \beta, j} \int_{0}^{\infty} d s\left\{\hat{d}_{\alpha}(s) \hat{d}_{\beta}^{\dagger} \hat{\rho}\left\langle\hat{j}_{\alpha}(s) \hat{j}_{\beta}^{\dagger}\right\rangle+\right.\text { H.c. } \\
& \left.+\hat{d}_{\alpha}^{\dagger}(s) \hat{d}_{\beta} \hat{\rho}\left\langle\hat{j}_{\alpha}^{\dagger}(s) \hat{j}_{\beta}\right\rangle+\text { H.c. }\right\} \\
& +\sum_{\alpha, \beta, j} \int_{-\infty}^{+\infty} d s\left\{\hat{d}_{\alpha}(s) \hat{\rho} \hat{d}_{\beta}^{\dagger}\left\langle\hat{j}_{\beta}^{\dagger} \hat{j}_{\alpha}(s)\right\rangle\right. \\
& \left.+\hat{d}_{\alpha}^{\dagger}(s) \hat{\rho}_{\beta}\left\langle\hat{j}_{\beta} \hat{j}_{\alpha}^{\dagger}(s)\right\rangle\right\} .
\end{aligned}
$$

(Note that there is no minus sign from fermion operator reordering in this factorization of dot and reservoir part, as the reservoir fermion operators are dragged past an even number of dot operators; compare, e.g., Ref. 41; alternatively, it is also possible to define them as commuting operators, since there is no interaction between them.) We get for the individual matrix elements (for brevity, the summation over $j$ $=l, r$ is implied):

$$
\begin{aligned}
\dot{\rho}_{++}= & -\rho_{++} \int_{-\infty}^{+\infty} d s\left\langle\hat{j}_{+}^{\dagger}(s) \hat{j}_{+}\right\rangle+\rho_{00} \int_{-\infty}^{+\infty} d s\left\langle\hat{j}_{+} \hat{j}_{+}^{\dagger}(s)\right\rangle \\
& -\rho_{-+} \int_{0}^{\infty} d s\left\langle\hat{j}_{+}^{\dagger}(s) \hat{j}_{-}\right\rangle-\text {H.c., } \\
\dot{\rho}_{+-}= & -\rho_{+-} \int_{0}^{\infty} d s\left\langle\hat{j}_{+}^{\dagger}(s) \hat{j}_{+}\right\rangle-\rho_{+-} \int_{0}^{\infty} d s\left\langle\hat{j}_{-}^{\dagger} \hat{j}_{-}(s)\right\rangle \\
& +\rho_{00} \int_{-\infty}^{+\infty} d s\left\langle\hat{j}_{-} \hat{j}_{+}^{\dagger}(s)\right\rangle-\rho_{++} \int_{0}^{\infty} d s\left\langle\hat{j}_{+}^{\dagger} \hat{j}_{-}(s)\right\rangle \\
& -\rho_{--} \int_{0}^{\infty} d s\left\langle\hat{j}_{+}^{\dagger}(s) \hat{j}_{-}\right\rangle .
\end{aligned}
$$

The equation for $\rho_{--}$follows from that for $\rho_{++}$by interchanging indices + and - .

Now we have to evaluate environment correlators, such as the prefactor of $\rho_{++}$in the second equation (e.g., for $j$ $=r)$,

$$
\left\langle\hat{r}_{+}^{\dagger} \hat{r}_{-}(s)\right\rangle=e^{i \varphi}\left\langle e^{-i \hat{\phi}} e^{-i \hat{\phi}(s)}\right\rangle\left\langle\hat{r}^{\dagger} \hat{r}(s)\right\rangle
$$

By introducing the bare tunneling rates $\Gamma_{R(L) 0}$ $=2 \pi D_{R(L)}\left\langle\left|t_{k}^{R(L)}\right|^{2}\right\rangle$ [compare Eq. (5)], we get, using Eq. (42) (remember $\hat{r}$ creates a reservoir electron),

$$
\left\langle\hat{r}^{\dagger} \hat{r}(s)\right\rangle=\frac{\Gamma_{R 0}}{2 \pi} \int d \epsilon\left[1-f_{R}(\epsilon)\right] e^{+i \epsilon s} .
$$

Here we have neglected any energy dependence of the tunnel-coupling and electrode density of states, assuming the relevant voltages and temperatures to be sufficiently small (but see below). The bath correlator in Eq. (B4) evaluates to $\exp \left[-\langle\hat{\phi} \hat{\phi}(s)\rangle-\left\langle\hat{\phi}^{2}\right\rangle\right]$, which can be expressed by using definition (12) for $P_{-}(\omega)$. There, we have to set $s \mapsto-s$ because of the reversed order in the $\hat{\phi}$ correlator,

$$
e^{-\langle\hat{\phi} \hat{\phi}(s)\rangle-\left\langle\hat{\phi}^{2}\right\rangle}=\int d \omega P_{-}(\omega) e^{i \omega s}
$$

Therefore, we obtain

$$
\int_{0}^{\infty} d s\left\langle\hat{r}_{+}^{\dagger} \hat{r}_{-}(s)\right\rangle=e^{i \varphi} \frac{\Gamma_{R 0}}{2} \int d \epsilon\left[1-f_{R}(\epsilon)\right] \widetilde{P}_{-}^{*}(-\epsilon),
$$

with

$$
\begin{aligned}
\widetilde{P}_{-}(\epsilon) & =\frac{1}{\pi} \int d \omega P_{-}(\omega) \int_{0}^{\infty} d s e^{i(\epsilon-\omega) s} \\
& =P_{-}(\epsilon)+\frac{i}{\pi} \int d \omega \frac{P_{-}(\omega)}{\epsilon-\omega} .
\end{aligned}
$$

The integral in the second line is understood as a principal-value integral. By using definitions (44)-(47) introduced in the main text, Eq. (B7) becomes equal to $\exp (i \varphi) \tilde{\gamma}_{R_{-}}^{*} / 2$. Other terms are evaluated similarly.

\section{APPENDIX C: CURRENT EXPRESSION FOR SEQUENTIAL TUNNELING THROUGH THE DOUBLE DOT}

At $T=0$, for the symmetric situation, the current $I$ is given by $I=e \gamma I_{0}\left[v, \delta_{L}, \delta_{R}\right]$, with

$$
\begin{aligned}
I_{0}\left[v, \delta_{L}, \delta_{R}\right]= & 2\left[-\delta_{L}^{2}+\left(v^{2}-1\right)\left(1+\delta_{R}^{2}\right)\right. \\
& \left.+2 \delta_{L} \delta_{R}\left(v^{2}-1\right) \cos \varphi+\delta_{L}^{2} v^{2} \cos ^{2} \varphi\right] \\
& \times\left[-3 \delta_{L}^{2}+2 \delta_{L} \delta_{R} v^{2}+\left(1+\delta_{R}^{2}\right)\left(v^{2}-3\right)\right. \\
& +2\left[v^{2}\left(1+\delta_{L}^{2}+\delta_{R}^{2}\right)+\delta_{L} \delta_{R}\left(v^{2}-3\right)\right] \cos \varphi \\
& \left.+\delta_{L}\left(\delta_{L}+2 \delta_{R}\right) v^{2} \cos ^{2} \varphi\right]^{-1}
\end{aligned}
$$


*Electronic address: Florian.Marquardt@unibas.ch

${ }^{1}$ P. Mohanty, E.M.Q. Jariwala, and R.A. Webb, Phys. Rev. Lett. 78, 3366 (1997).

${ }^{2}$ P. Cedraschi, V.V. Ponomarenko, and M. Büttiker, Phys. Rev. Lett. 84, 346 (2000).

${ }^{3}$ P. Cedraschi and M. Büttiker, Phys. Rev. B 63, 165312 (2001); Ann. Phys. 289, 1 (2001).

${ }^{4}$ A.D. Zaikin and D.S. Golubev, Physica B 280, 453 (2000).

${ }^{5}$ F. Marquardt and C. Bruder, Phys. Rev. B 65, 125315 (2002).

${ }^{6}$ K.E. Nagaev and M. Büttiker, Europhys. Lett. 58, 475 (2002).

${ }^{7}$ F. Guinea, Phys. Rev. B 65, 205317 (2002).

${ }^{8}$ D.S. Golubev, G. Schön, and A.D. Zaikin, cond-mat/0208548, 2002 (unpublished).

${ }^{9}$ Y. Imry, cond-mat/0202044, 2002 (unpublished).

${ }^{10}$ A. Stern, Y. Aharonov, and Y. Imry, Phys. Rev. A 41, 3436 (1990).

${ }^{11}$ J. König and Y. Gefen, Phys. Rev. Lett. 86, 3855 (2001); Phys. Rev. B 65, 045316 (2002).

${ }^{12}$ T. Fujisawa, T.H. Oosterkamp, W.G. van der Wiel, B.W. Broer, R. Aguado, S. Tarucha, and L.P. Kouwenhoven, Science 282, 932 (1998).

${ }^{13}$ T.H. Stoof and Y.V. Nazarov, Phys. Rev. B 53, 1050 (1996).

${ }^{14} \mathrm{Ph}$. Brune, C. Bruder, and H. Schoeller, Phys. Rev. B 56, 4730 (1997); Physica E (Amsterdam) 1, 216 (1997).

${ }^{15}$ R. Ziegler, C. Bruder, and H. Schoeller, Phys. Rev. B 62, 1961 (2000).

${ }^{16}$ T. Brandes and B. Kramer, Phys. Rev. Lett. 83, 3021 (1999).

${ }^{17}$ R. Aguado and L.P. Kouwenhoven, Phys. Rev. Lett. 84, 1986 (2000).

${ }^{18}$ S. Debald, T. Brandes, and B. Kramer, Phys. Rev. B 66, 041301 (2002).

${ }^{19}$ M. Keil and H. Schoeller, cond-mat/0205308, 2002 (unpublished).

${ }^{20}$ H. Akera, Phys. Rev. B 47, 6835 (1993).

${ }^{21}$ T.V. Shahbazyan and M.E. Raikh, Phys. Rev. B 49, 17123 (1994)

${ }^{22}$ A.W. Holleitner, R.H. Blick, A.K. Hüttel, K. Eberl, and J.P. Kot- thaus, Science 297, 70 (2002).

${ }^{23}$ D. Boese, W. Hofstetter, and H. Schoeller, Phys. Rev. B 64, 125309 (2001).

${ }^{24}$ D. Loss and E.V. Sukhorukov, Phys. Rev. Lett. 84, 1035 (2000).

${ }^{25}$ M.-S. Choi, C. Bruder, and D. Loss, Phys. Rev. B 62, 13569 (2000).

${ }^{26}$ R. Aguado and D.C. Langreth, Phys. Rev. Lett. 85, 1946 (2000);

R. Lopez, R. Aguado, and G. Platero, ibid. 89, 136802 (2002).

${ }^{27}$ D. Boese, W. Hofstetter, and H. Schoeller, Phys. Rev. B 66, 125315 (2002).

${ }^{28}$ K. Haule and J. Bonca, Phys. Rev. B 59, 13087 (1999).

${ }^{29}$ L.G. Mourokh, N.J.M. Horing, and A.Yu. Smirnov, Phys. Rev. B 66, 085332 (2002).

${ }^{30}$ E. Buks, R. Schuster, M. Heiblum, D. Mahalu, and V. Umansky, Nature (London) 391, 871 (1998).

${ }^{31}$ I.L. Aleiner, N.S. Wingreen, and Y. Meir, Phys. Rev. Lett. 79, 3740 (1997).

${ }^{32}$ G.-L. Ingold and Y.V. Nazarov, in Single Charge Tunneling, Vol. 294 of NATO Advanced Study Institute, Series B: Physics, edited by H. Grabert and M. Devoret (Plenum, New York, 1992).

${ }^{33}$ G. Schön, T. Dittrich, P. Hänggi, G.-L. Ingold, B. Kramer, G. Schön, and W. Zwerger, Quantum Transport and Dissipation ( Wiley-VCH, Weinheim, 1998).

${ }^{34}$ G.D. Mahan, Many-Particle Physics (Plenum, New York, 1981), pp. 269-310.

${ }^{35}$ M.H. Devoret, D. Esteve, H. Grabert, G.L. Ingold, H. Pothier, and C. Urbina, Phys. Rev. Lett. 64, 1824 (1990).

${ }^{36}$ P. Minnhagen, Phys. Lett. A 56, 327 (1976).

${ }^{37}$ V.F. Gantmakher and Y.B. Levinson, Carrier Scattering in Metals and Semiconductors (North-Holland, Amsterdam, 1987), Sec. 3.5.

${ }^{38}$ K. Blum, Density Matrix Theory and Applications (Plenum, New York, 1996).

${ }^{39}$ H.-A. Engel (private communication).

${ }^{40}$ Y. Meir and N. Wingreen, Phys. Rev. Lett. 68, 2512 (1992).

${ }^{41}$ H. Schoeller, Lect. Notes Phys. 544, 137 (2000). 